\title{
Efficient computational techniques for mistuning analysis of bladed discs: a review
}

\author{
Jie Yuan ${ }^{1,5}$, Fabrizio Scarpa ${ }^{1,3}$, Giuliano Allegri ${ }^{2}$, Branislav Titurus ${ }^{1,3}$, Sophoclis Patsias ${ }^{4}$, \\ Ramesh Rajasekaran ${ }^{4}$ \\ ${ }^{1}$ Aerospace Engineering, University of Bristol, BS8 ITR Bristol, UK \\ ${ }^{2}$ Department of Aeronautics, Imperial College London, London SW7 2AZ, UK \\ ${ }^{3}$ Dynamics and Control Research Group, University of Bristol, BS8 1TR Bristol, UK \\ ${ }^{4}$ Rolls-Royce plc, PO Box 31, DE24 8BJ Derby, UK \\ ${ }^{5}$ Aerospace Division, Cranfield University, MK430AL, UK
}

\begin{abstract}
This paper describes a review of the relevant literature about mistuning problems in bladed disc systems, and their implications for the uncertainty propagation associated to the dynamics of aeroengine systems. An emphasis of the review is placed on the developments of the multi-scale computational techniques to increase the computational efficiency for the linear mistuning analysis, especially with the respect to the reduced order modeling techniques and uncertainty quantification methods. The non-linearity phenomena are not considered in this paper. The first two parts describe the fundamentals of the mechanics of tuned and mistuned bladed discs, followed by a review of critical research efforts performed on the development of reduced order rotor models. The focus of the fourth part is on the review of efficient simulation methods for the stochastic analysis of mistuned bladed disc systems. After that, we will finally provide a view of the current state of the art associated to efficient inversion methods for the stochastic analysis, followed by a summary.
\end{abstract}




\section{Content}

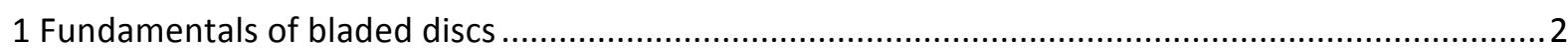

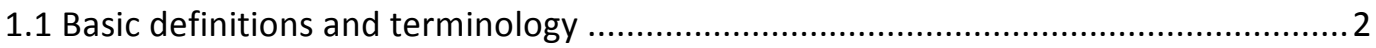

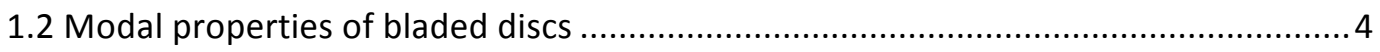

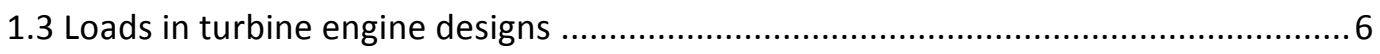

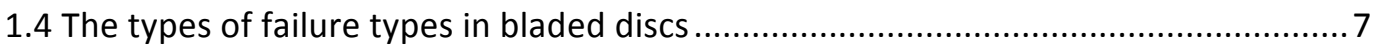

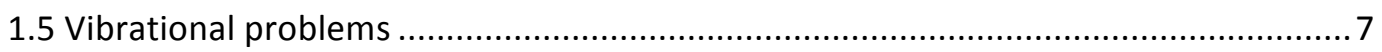

1.6 Strategies to suppress vibration in aeroengine rotor systems ................................ 9

2 Fundamental aspects for mistuned bladed disc system .......................................................... 11

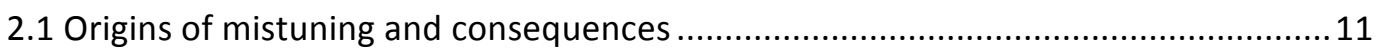

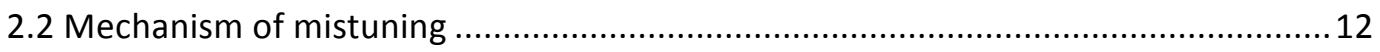

2.3 Mistuning problems in open literature ............................................................... 14

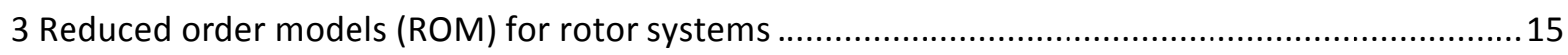

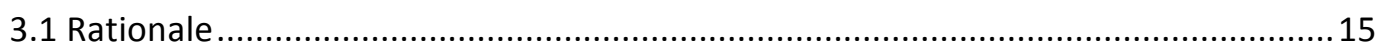

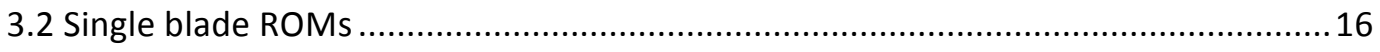

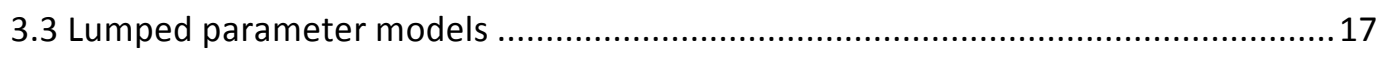

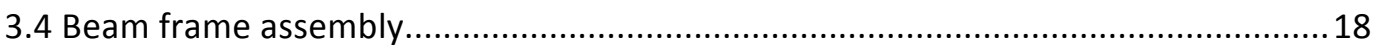

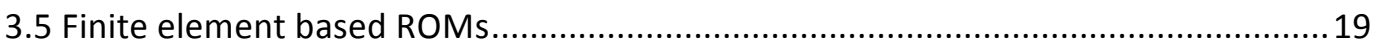

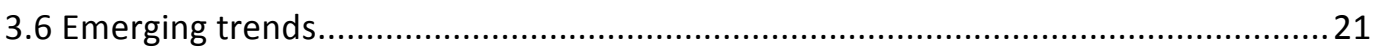

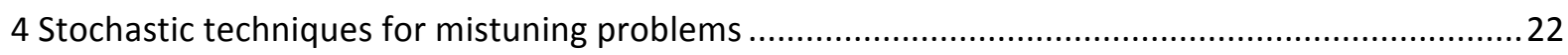

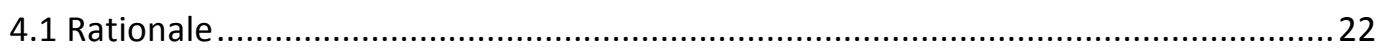

4.2 Current stochastic methods used in mistuning ..................................................22

5 Efficient inversion methods for the stochastic analysis............................................26

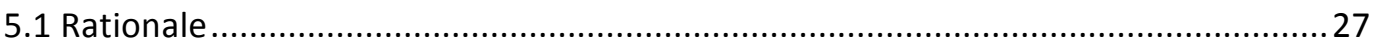

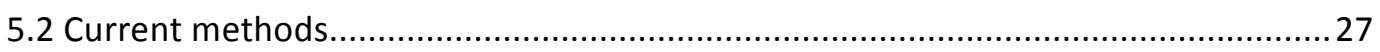

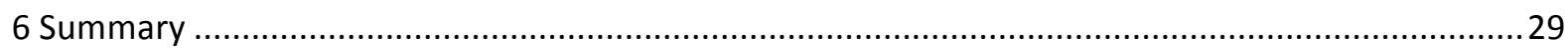




\section{Fundamentals of bladed discs}

The objective of this section is to give the readers a brief introduction to bladed discs systems in aeroengines. The basic definitions and terminology related to bladed discs and their modal properties are firstly described. Then, we will briefly introduce an account of the loading occurring on bladed discs in aeroengines, as well as the resulting vibrational problems and implications. A review of the current strategies to suppress vibrations in bladed disc systems problems is also presented.

\subsection{Basic definitions and terminology}

A bladed disc is usually composed by a disk with a relatively large number of blades assembled in its circumference. A bladed disc is a typical example of a cyclically periodic structure, in which a cyclic-symmetric sector repeats itself around the axis of rotation (Figure 1). In gas turbine engines, dovetail root fixings are frequently used to attach the blades to the disk. Under aerodynamic loading the surfaces of the blade and the disc can therefore come into contact with each other either at a point, or along a line or a surface, or a combination of those. The contact region transmits the forces from one body to the other by means of normal compressive stresses, and tangential or shear stresses when friction is present. In an axial flow compressor, bladed discs are commonly mounted on a rotating shaft, together with a stator fixed to the casing. All the blades assembled in either the rotors or the stators are aerodynamically profiled to generate a pressure difference across the stages. In order to create sufficient thrust, the bladed discs are commonly subjected to very severe mechanical stresses and vibrations, due to the unsteady aerodynamic forces originated from the relative motions of the non-rotating and rotating parts at speeds between 10,000 to 17,000 rpm. These large and periodic loadings may result in high-cycle fatigue (HCF) failure, or create large plastic deformations in the blades or the whole assembly [1].
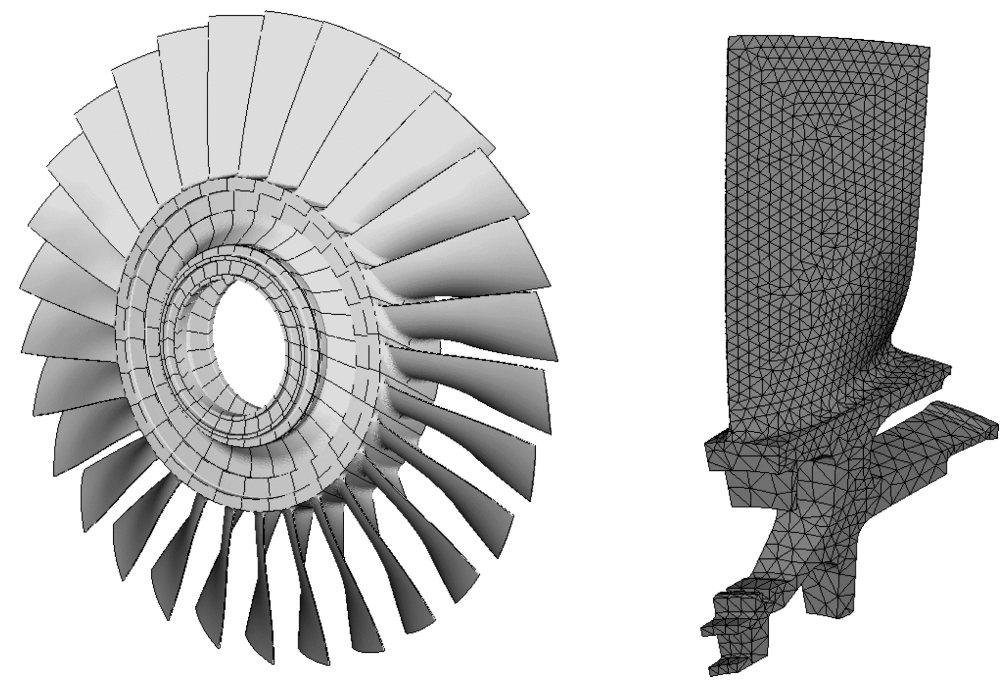

Figure 1 A high pressure compressor and one of its blades components [2] 
Depending on the type of application, blades are assembled around the disk in various ways. Their configurations can be with shrouds, which can increase the blade stiffness and also provide the so-called inter-blade coupling. Figure 2 shows an example of a bladed disc with shrouds. From an aeroelastic point of view, shrouds can also be used to enhance the stability of the system because they can be tailored to avoid flutter caused by the interaction between aerodynamic loadings, inertias and the elasticity of the structure itself. In bladed discs without shrouds neighbouring blades are coupled through the disk itself, and the degree of the inter-coupling depends on the flexibility of the disk. Inter-blade coupling is in general an important parameter for the dynamic performance of mistuned bladed discs [3].

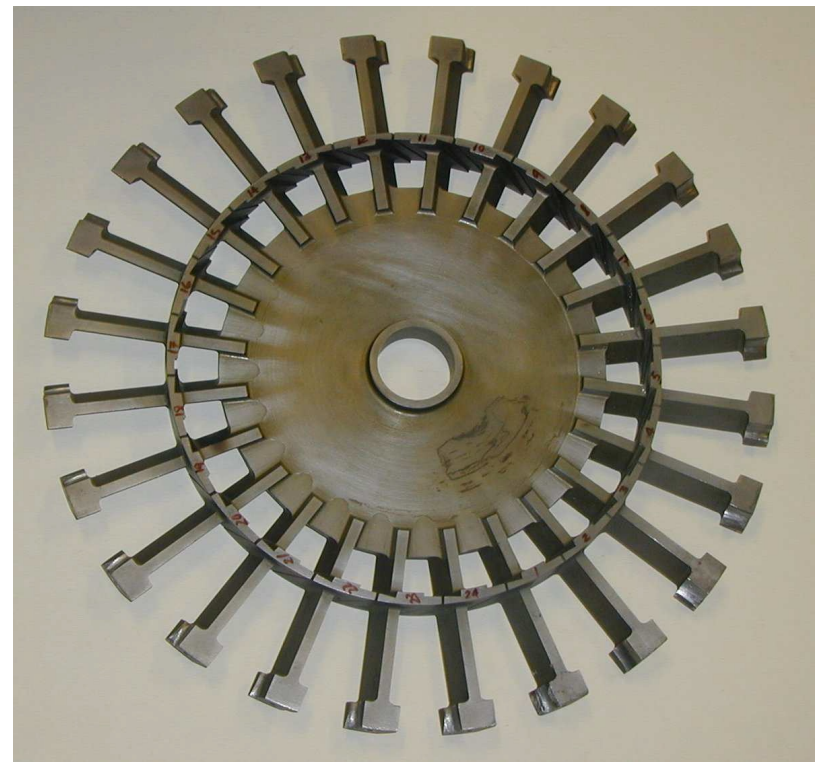

Figure 2 A bladed disc with shrouds [4]

Compared to classical bladed disc systems, a blisk has blades that are an integral part of the system. The blades and the disc are manufactured together as a whole structure. The use of blisks in aero-engines can potentially reduce significantly the weight, the aerodynamic losses and the number of the parts leading to a significant increase in efficiency and decrease of the complexity of the system [5]. The use of blisks can also significantly eliminate the presence of contact stresses otherwise existing in classical blades systems, and therefore alleviate HCF and low-cycle fatigue (LCF) problems, increase the fundamental frequencies and lower the vibrational and centrifugal loads during service. Moreover, blisks can minimize the chances of blades leaving the disc due to high centrifugal stresses and the rubbing of their tip against the casing. Blisks however present some disadvantages for gas turbine engines applications. One of their main drawbacks being that once one of the blades fails the entire blisk assembly has to be replaced, with detrimental consequences for the operational and maintenance costs. Furthermore, the manufacturing of a blisk is more challenging and expensive, as conventional manufacturing approaches used in turbine discs are not suitable any more [5]. 


\subsection{Modal properties of bladed discs}

Bladed disks exhibit well-defined vibrational mode shapes because of their cyclic symmetry [6]. The eigenmodes in a bladed disk occur either as double modes (two identical natural frequencies with their mode shapes circumferentially separated by a $90^{\circ}$ phase shift) or single modes (all the blades experience the same amplitude). The double modes can be described as sinusoidal variations of the circumferential displacement around the bladed disk, which can be expressed in the form of $\sin (n \theta+\psi)$, where $\theta$ is a inter blade angle, $\psi$ is a phase angle and $n$ is the number of nodal diameters (ND) [6]. Assuming $\mathrm{N}$ is the number of the blades in a bladed disc, the number of ND is defined as the number of lines with zero displacement across the assembly. It can be equal to any integer between 0 and $N / 2$ (if $N$ is even) or $(N-1) / 2$ (if $N$ is odd). However, the nodal diameter can be higher than $N / 2$ only when discreet points do not describe the circumferential motions of the continuous disk. The single modes only occurs when $n=0$ (in phase mode) and when $n=N / 2$ or $(N-1) / 2$ (out of phase mode), while all the other modes appear in pairs. The example of the rotor mode with different number of ND is shown in Figure 2.1. However, it is worth noticing that for a tuned bladed disc the angular orientation of these nodal diameters is not fixed, and it can be aligned along arbitrary positions in the structure. The mode shapes of a disk with the same nodal diameter number may possess nodal points in the radial direction in addition to the nodal diameter [4]. The circles formed by these points around the bladed disk are called nodal circles, an example of which can be observed in Figure 3 (a).

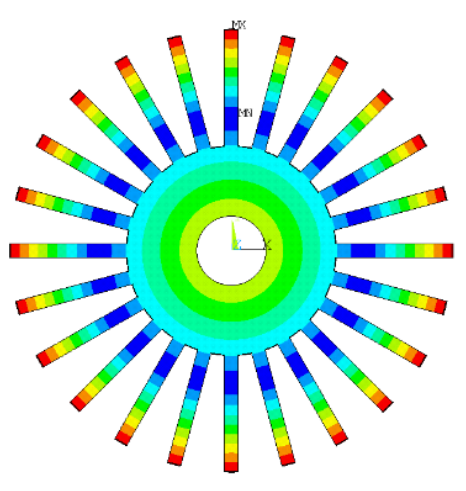

(a)

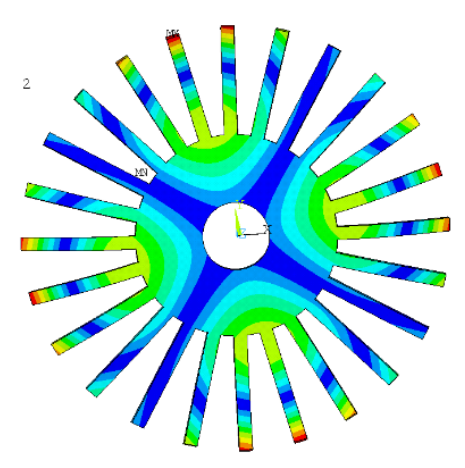

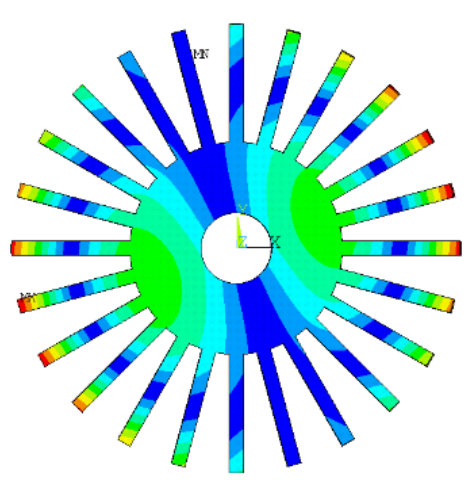

(b)

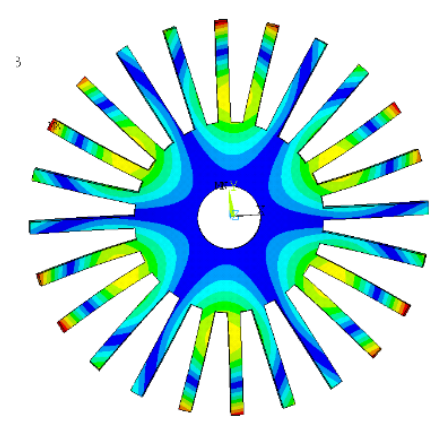


Figure 3 Examples of the rotor modes with modal displacement sums for ND numbers of (a) 0 (b) 1 (c) 2 (d) 3

Figure 4 shows the typical variations of the natural frequencies (NFs) against the NDs of the finite element model that characterizes the free vibration of an industrial bladed disc shown in Figure 1. The fact that the disc itself becomes more rigid with the increasing NDs makes the NFs of the rotor gradually increase and finally approach the NFs of the blade alone. The horizontal lines at high NDs represent the blade-dominated modes, while the slope-varying lines at low NDs represent disk-dominated ones. With the increase of the nodal diameters, the NFs in each family asymptotically approach one of blades frequencies (i.e., with the blade fixed at its interface with the disk). These modes also referred to cantilevered blade modes and include flexural bending (F), torsion (T), stripe (S), chordwise bending (C) and edgewise bending (E) families [7]. Examples of three fundamental modes, namely, first \& second bending and first torsion modes, are shown in Figure 5. Frequency veering phenomenon commonly occurs at low NDs. In field of the structural dynamics, the frequency veering refers to the loci of the eigenvalues, such as natural frequencies, of a dynamic system converging and then veering apart without crossing when the eigenvalues are plotted as a function of some physical parameter [8]. The veering regions in rotor dynamics shows in Figure 2.4 that indicate some significant disk-blade interactions. They have an important effect on the forced frequency response of mistuned systems. From Figure 4, it is possible to observe that the $T$ and S modes exhibit significant variations of the NFs within specific intervals of the NDs. This suggests the existence of inter-blade coupling in these modes that facilitates the energy transfer between the blades through the disc. Therefore, this mechanism can generate mode localization and extremely high forced frequency responses.

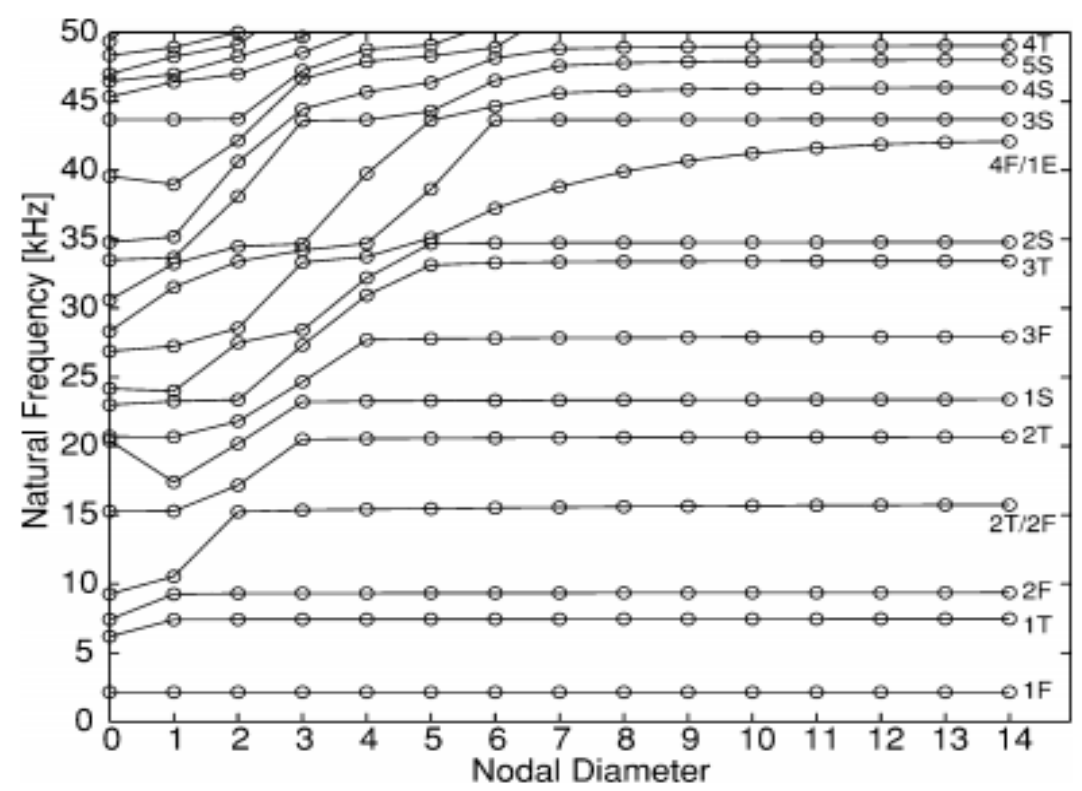

Figure 4 Typical variations of natural frequencies with nodal diameters in a tuned bladed disk [7] 


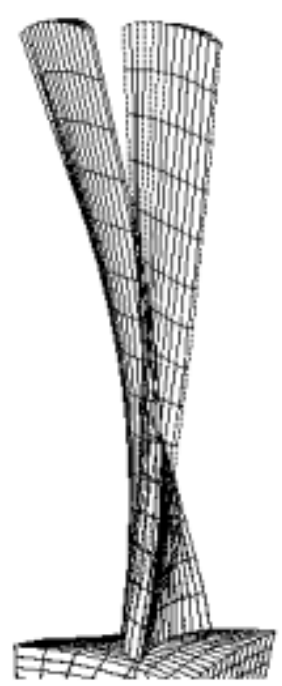

(a)

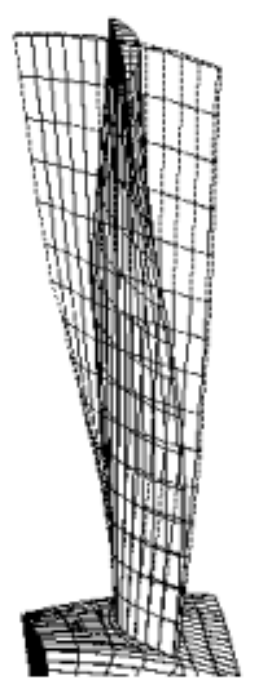

(b)

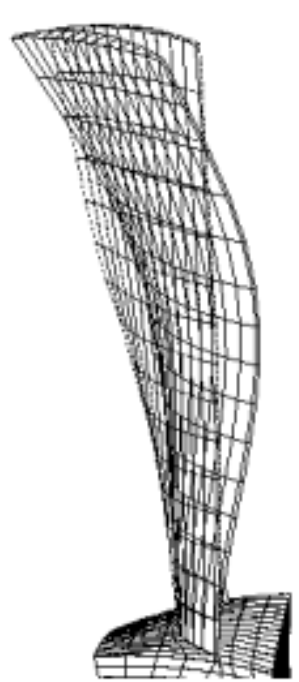

(c)

Figure 5 Example of the mode shapes of a fan blade (a) First bending (1F) mode (b) First torsion (1T) (c) Second bending (2F) [7]

\subsection{Loads in turbine engine designs}

In gas turbine compressors temperature and stresses are the two dominant factors that affect adversely the operational life of the bladed discs. The variation of the gas temperature during the operation leads to an alternate cycle of stresses and strains on the blades and the disc. The range of the working cycle temperature in a high-pressure compressor is usually between $200 \mathrm{~K}$ and $700 \mathrm{~K}$. The highest temperature is limited to the endurance of titanium alloys that are traditionally used in the compressor. The latest type of titanium alloys have good properties up to about $770 \mathrm{~K}$ [9]. The thermal stress levels from the cyclic loads make the material to behave frequently within the plastic deformations range and subjected to particular rates of creep, leading to potential onsets of early fatigue damage. The creep elongation would be also accumulated with time and the original tip gap between the blade and casing is gradually reduced, which may result in hard rubbing and structural failure. As a result, proper material treatment and coating layer should be adopted on the blades.

High stress levels can also be generated from steady or unsteady aerodynamic loads. The sources of the dynamic loading present in aero-engines can be mainly classified into three types. The most common type is the unbalanced time varying aerodynamic force, which is represented by a vector relative to the stationary casing and rotating at a frequency equal to the speed of the turning shaft [10]. The second source of excitation perhaps the most common one is the so-called engine order excitation that comes from the imperfect axis-symmetry of the non-oscillatory gas flow when it is passing through the engine. The asymmetry is due to the non-uniform fluid stream in the intake and the subsequent discontinuities in the geometry 
of the aeroengine, such as bearing supports, nozzle guide vanes and combustion chambers. The intensity of the pressure exerted by the axial flow on the blades is therefore non-uniform around the circumferential direction at each section of the engine. The non-uniform loading on the downstream rotating blades is felt as a time-varying pressure and therefore generates a dynamic response of the rotating bladed discs[10]. The third type of the aerodynamic loads is originated from the turbulent flow of the external environment. However, it does not affect rotating blades in a significant way, and therefore it is not considered as one of the major dynamic loads acting in aeroengine components [10].

\subsection{The types of failure types in bladed discs}

Bladed discs may still experience some unexpected mechanical failures during their operational service even with the use of careful design and advanced manufacturing techniques. These failures are very likely to occur at excessive stress levels when the bladed discs are in overload situations. The resulting high stress levels could lead to yielding of the components in a disc assembly, with permanent plastic deformations. LCF can also lead to the unexpected failure of the blades. In gas turbine engines these situations occur when the stress levels reverses under some typical loading conditions, such as engine restart. Moreover, tip rubbing between the blade and the casing is also an important potential source of mechanical failure and loss of performance, and the effective design and control of the tip tolerance during operations is critically important. The worse type of failure for rotating rotors is the blade-off situation during over-speeding. Blade-off has to be avoided by proper design of the joints and by adjusting the rotating speed margins. However, the majority of the failures in disc-bladed systems derives from high cycle fatigue, which is responsible for up to $70 \%$ of all failures occurring in gas turbine engines [11]. High cycle fatigue has historically led to the premature failure of major aero engine components and in some cases even the loss of the engine and the entire aircraft. These failures are mainly because of vibration related problems under high-speed aerodynamic loads. Bladed discs may also suffer from unstable vibrations such as flutter and rotor dynamics instability, which potential dramatic consequences.

\subsection{Vibrational problems}

The dynamic deformation or induced stress of a vibrating structure can be significantly different to the correspondent static response, even when the same maximum amplitude of the load is considered [10]. In gas turbine engines dynamic loads can induce stress levels 100 or 1000 times higher than their static counterpart, with consequent onset of failure and high cycle fatigue. Dynamic loads would also result in malfunctions of devices and levels of discomfort noise. For rotating components like bladed disk assemblies, the loss of structural integrity tends to be more critical than in stationary parts like casting, pipework because the consequences of even a small failure on the rotating part can be magnified by unbalanced forces and secondary damage by potential debris being thrown off [1]. Vibration problems therefore give rise to critical issues in aeroengine design and manufacturing. 
Blades discs are particularly prone to dynamic loadings. The major source of vibration is engine order excitation, which makes assemblies of blades experience periodic response changes in time and phase variation between blades. When an assembly of $N$ blades is being subjected to an excitation of engine order $k$ the inter-blade phase angle is $2 \pi k / N$. The excitation of order $k$ can only excite those modes with $k$ nodal diameters for a perfectly tuned bladed disk, or having a $\mathrm{k}^{\text {th }}$ nodal diameter component in a case of a mistuned bladed disk. However, the modes with $k$ nodal diameters can also be excited by engine order $N-k$ and $N+k$ because of the spatial aliasing of the rotor [7]. The engine order of downstream flow commonly depends on the features of the aeroengine architecture such as number of stages, blades or stators. In the gas turbine industry, the Campbell diagram (natural frequencies versus the rotational speed or engine order) is commonly used to describe the dynamic characteristics of bladed disc systems.

If an extremely stiff disc is part of a bladed disk assembly, the single blades could be considered as isolated parts for dynamic analysis purposes. Under dynamic loads one of the most critical stress locations in an assembly is at the attachment root of the blades as shown in Figure 6. Although the joining mechanism between the blade root and the disc is represented by interference, the difference of thermal expansion coefficients between the interface materials introduces some flexibility into the system during the operation. Due to the high levels of contact stress, the materials present in the dovetail also can be deformed. Even when no obvious macroscopic motion occurs, some degree of micro-slip motion would occur at the edges of the interface under the dynamic deformation of the blade. The existence of stick and micro slipping zones at the blade root surfaces allows local slipping at the extremities of the contact zone [12]. The positive effect of this micro slip in the dovetail is to contribute to dissipate energy for the reduction of the vibrational amplitude of the blades, however it also gives rise to fretting between the blade root and the disc. As a result, bladed disc interfaces experience a combination of low cycle fatigue (LCF) due to major loading events, and high cycle fatigue (HCF) due to minor (amplitude) dynamic loads [13]. LCF and HCF loading combinations may result in the early fatigue life of the blades, as much as $40 \%$ to $60 \%$ lower along with the crack initialization at the edges of the contact zone[14]. Moreover, bladed discs may also be subjected to unstable vibrations, which may lead to dramatic consequences like blade flutter or rotor instability with divergence [15]. Instability can also occur in the presence of excessive mechanical damping in a rotor when there is insufficient external damping from the bearings and working fluid [10]. 


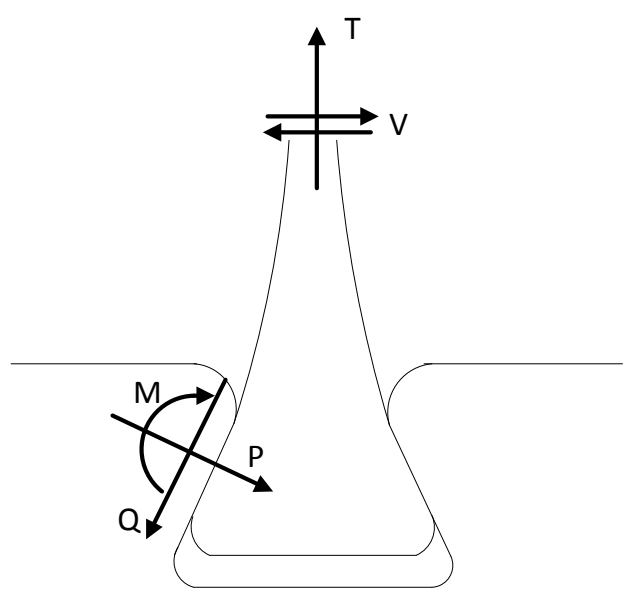

Figure 6 Free body diagram of dovetail joint (T: Centrifugal force; V:vibratory loading on the blade; Q:Contact frictional stress; P: Contact normal stress; M: Bending moment) [16]

The vibration measurement therefore is necessary to monitor and diagnose the conditions of bladed discs. This could allow the early detection of blade damage in order to avoid the unrepairable situation. The traditional approach consists of using a telemetric strain-gauge system where the strain gauges are attached at reference points of selected blades. The frequencies and amplitude of blade vibrations can be obtained through the measurement of strain dynamic components. However, the method would not be able to gain the diagnostic information of the static positions of the blades, such as the root of the blade. Also, the lifetime of the strain gauge is short due to high speed of the rotation and large temperature variation. These strain gauges are only able to survive days or weeks [17]. Therefore, non-contact systems with stator sensors based on the tip-timing method has been developed. The blade-tip timing (BTT) techniques can be used to identify vibrational problem of all the blades through the measurement of the passing times of blade tip under stationary points. Although the measurement cost of BTT approach is much lower than strain-gauge approach, the recovery of the vibration information from BTT methods is complex. It is mainly because that the analysis techniques differ for various type of blade response being sampled. In the case of synchronous vibrations for a single mode, single or two parameter techniques can be used to analyse the BTT data. By using autoregressive methods, BTT analysis can recover the correct frequencies when two blade modes are excited simultaneously by a synchronous vibration. The current progress shows that BTT method can be potentially used to monitor blade vibrations of tuned and mistuned bladed discs. More details of the BTT techniques can be referred to [17-19]

\subsection{Strategies to suppress vibration in aeroengine rotor systems}

The primary approach to control vibrations in bladed discs is to avoid critical resonances at engine speeds corresponding to operational conditions during lengthy periods of time. These critical conditions can be avoided by ensuring that crossings of the natural frequencies in the Campbell diagram do not exist within the running speed range. For that purpose, designers 
have either the option of changing the operational speed, or to modify the natural frequencies of the bladed disks. Considering the overall design constraints existing in modern gas turbines, the latter option is considered more achievable [10]. However, the modes in a bladed disc system within in the range of the rotating speed is in general very dense, and it is often not feasible to adjust the dimensions of the components to avoid critical resonances. Therefore the dimensions and parameters of the disc system belonging to the baseline design must be controlled to avoid severe wear or damage. The way to achieve a minimization of the vibration response close to resonance is by introducing damping into the system, so that the peak value of the dynamic response of the blade can be reduced to safe levels. Figure 7 shows some examples of damping treatments and solutions being used to increase the vibrational performance of the bladed disc.

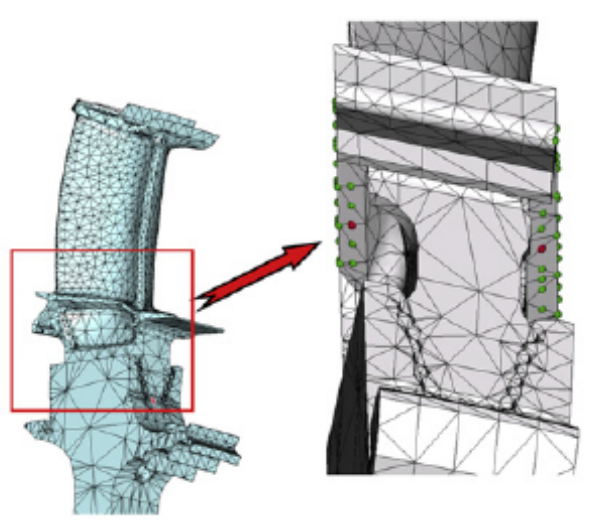

(a) Dry friction: underplatform damper [10]

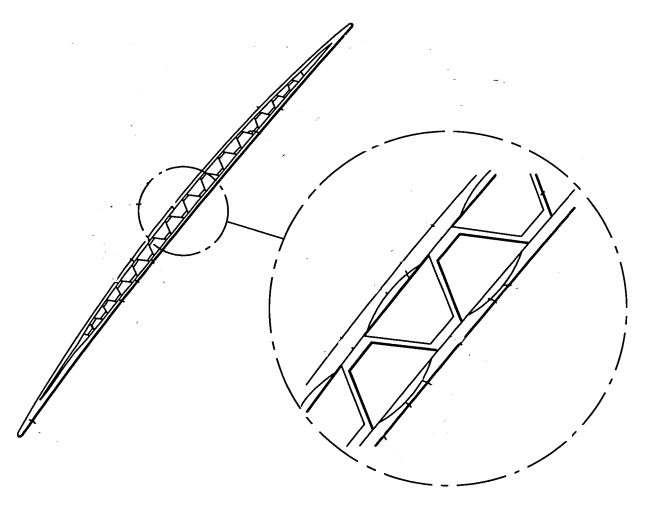

(c) Internal damping with membrane precursor [20]

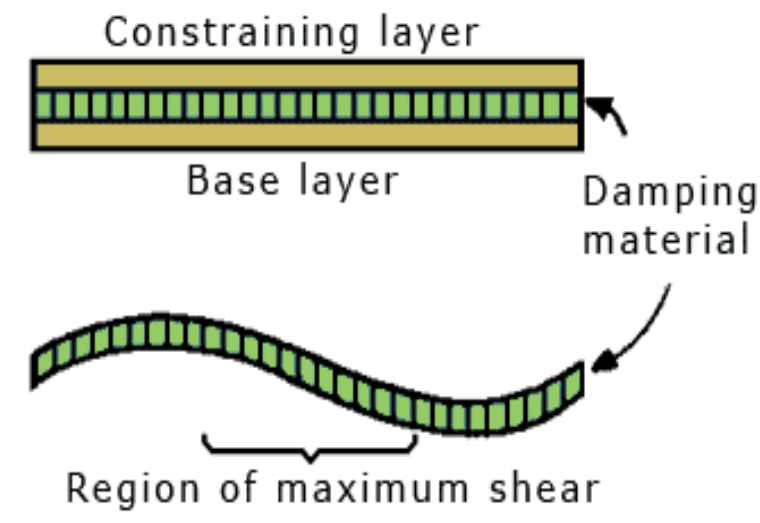

(b) Constraint layer on the surface [23]

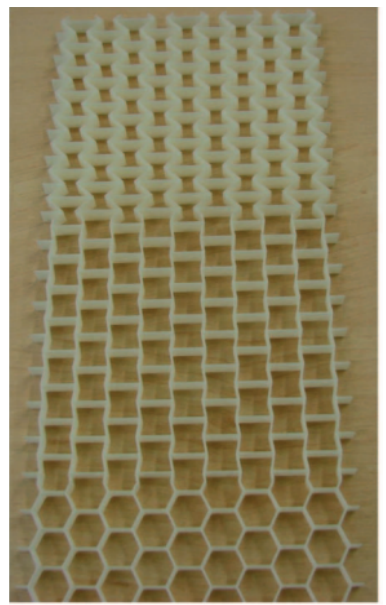

(d) Novel gradient core blade configuration [21]

Figure 7 Examples of approaches to increase vibrational performance of the bladed disc

Dry friction is still used as the principle damping mechanisms in bladed disc systems [22]. The rationale behind the use of friction damping is to make the mating interfaces to fret against each other in a controlled way and therefore to dissipate efficiently the vibrational energy. The main forms of using friction damping in bladed discs is via root damping (the 
relative slip between the blade root and disc in the dovetail), tip damping (the slip between the shrouds, or between the tip of the blade and the casing) and under platform dampers as shown in Figure 7 (a). The first two techniques are the damping sources that follows from the adoption of standard aeroengine component designs, while the third type can be a designed-in device used to provide significant damping for potentially harmful resonances [10].

The damping of blades can also be enhanced by using several passive surface treatments, such as aerodynamic membrane and constraint layer damping [23]. The internal damping mechanism is also introduced into bladed discs by face-to-face contact with a membrane precursor [20]. The use of special post-processing techniques on the blades surfaces like plasma coating is also effective to increase the resistance of a bladed disc to vibrations [24]. In addition, the dynamic response of the blades close to resonance can also be reduced up to $20 \%$ by using the aerodynamic mistuning technique, which consists in a deliberate mistuning of the dynamic pressure pattern by including (or removing) few more stator blades or adjusting the gap between the blades in the downstream flow [25]. This method can also be used to avoid in an effective manner special aerodynamic conditions that make flutter possible in running speeds. It is also possible to improve the vibrational performance of a bladed disc by using novel core blade configurations like gradient cellular auxetic topology or composite structures that provide the tailoring of the stiffness distribution along the blade and reduce the modal displacements [21, 26, 27]. Active devices are also used to reduce vibrational levels, such as active magnetic bearings that generate adaptive opposite dynamic forces to the external dynamic loading under operating conditions [28].

\section{Fundamental aspects for mistuned bladed disc system}

In this section, we will review the origins of the mistuning phenomenon in bladed discs and how it affects the dynamic behavior of the bladed discs. Then, an overview of the previous research efforts on solving mistuning problems will be given.

\subsection{Origins of mistuning and consequences}

Typically, a bladed disk consists in a set of disk-blade sectors that are designed to be identical. However, there are inevitable tiny variations in the structural properties of individual blades from manufacturing tolerances, materials dispersions or geometric non-uniformity, operational wear and tear and non-uniform assembly of the blades [29]. These variations will lead to the deviation of the blades' NFs from their nominal design value, which is called mistuning. The research on the origins and solutions of mistuning in bladed discs was started by Armstrong in 1955 [30] and Tobias and Arnold in 1957 [31]. Whitehead in 1966 focused on the influence of mistuning on the forced vibration behavior of bladed systems [32], followed on the topic by Ewins [33] and Dye \& Henry in 1969 [34]. The mistuning problem 
has been an obvious focus of engineers from the world leading aero engine manufacturers, such as Rolls-Royce, PW and GE, for more than 50 years [35]. It is so because of mistuning that vibrational levels may be more than 5 times higher than the counterpart in a tuned bladed disc. The amplitude factor term was introduced in previous research to measure the severity of the consequences of the mistuning response levels, being defined as the ratio of the highest forced vibration response level found in a given mistuned bladed disc to the highest forced vibration response level of the tuned counterpart under the same excitation pattern [36]. Another design factor related to mistuning is the adjusted amplitude factor proposed by Lim [37], which is expressed as the ratio between the peak response level of any given blade on a mistuned bladed disc to the peak forced level of a cantilevered blade with the same properties and type of excitations. This factor is mainly used in robust design since it is independent of the disc configuration [36].

Due to the high rotors speeds in gas turbine engines a high level of dynamic response from one of the blades could significantly reduce the HCF life of the whole bladed disc, with negative effects on the durability and reliability of the engine. The costs associated to high cycle fatigue-related problems in aeroengine was reported in 2002 to be around $\$ 400$ million each year, which corresponds to $30 \%$ of all jet engine maintenance costs [38]. However, one positive aspect of mistuning is that it is generally believed to be beneficial for flutter problems [10], and therefore mistuning could be effectively used to avoid some classes of unsteady vibrations.

\subsection{Mechanism of mistuning}

In a mistuned bladed disc, the relationship between the amplitude factor and its dependent variables can be described by an amplitude factor function with input parameters being the blade and disc design parameters, the mistuning and the excitation patterns. Design parameters for a bladed disc usually includes the number of blades, the inter-blade coupling and damping levels. The effect of the number of blades $N$ on the maximum amplitude factor is initially expressed by the Whitehead Factor [32]:

$$
A_{W F}=\frac{1}{2}(1+\sqrt{N})
$$

Equation (2.1) may however overestimate the effect on the amplitude factor given by the blade number [39]. A mistuning pattern is defined as the sequence of mistuning parameters present on a mistuned bladed disc. The mistuning parameter usually refers to the fractional difference between the NF of a mistuned blade with the tuned (baseline) counterpart frequency. The excitation pattern commonly refers to the engine order excitation. In a forward-rotating bladed disc, the engine order excitation can be approximated to a backward travelling excitation pattern over a stationary one, with an equivalent frequency of the engine order multiplied by the rotation speed [36].

Figure 8 shows a flowchart of how the mistuning phenomenon may result in higher dynamic responses in a bladed disc. The uncertainties associated to the structural, material, 
manufacturing and assembly characteristics of a bladed disc significantly affect the modal properties of the original tuned system. The natural frequencies of the circumferential double modes are split and the mode shapes distorted [33, 35, 40-42]. Simultaneously, the new mode shapes of the mistuned system contain multiple harmonic contents rather than a single one, therefore that they can be excited by multiple engine orders at the same time [7]. The presence of mistuning would lead to mode localization, in which the mode shapes undergo dramatic changes to become strongly localized and the kinetic energy in blades transferred and confined to only one or few blades [42]. Mode localization may result in excessively larger dynamic amplitudes compared to the one of a tuned system [43, 44]. However, the mistuning phenomenon would not always give rise to a higher response, and in some cases may even contribute to a much lower overall dynamic response. Hodge showed that the mode localization of a bladed disc becomes worse either with increasing mistuning levels, or by decreasing the mechanical coupling between blades [40]. However, the extent of mode localization is not always relevant to the maximum forced response and very much depends on the mistuning levels of the blades and their mistuning patterns. Considering the demanding safety and airworthiness regulations in the civil aviation industry, the consequences of worst case scenarios have to be taken into account during the design phase of the engine.

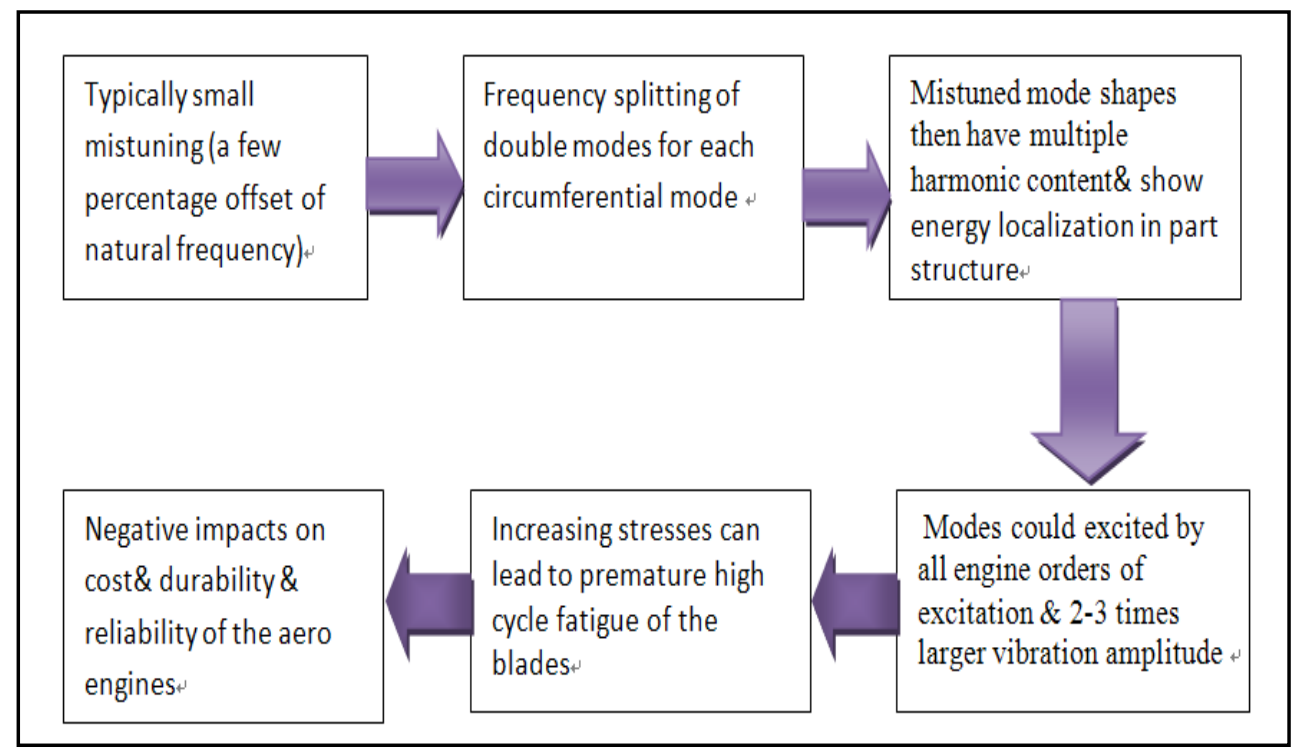

Figure 8 A flowchart showing the mechanism about how to the mistuning affects the dynamic response of a bladed disc system.

References [33, 45] indicate that the maximum amplitude of the blade dynamic response increases with increasing mistuning only up to a certain level, after which mistuning leads to lower forced response amplitudes. Figure 9 shows the variation of the $99^{\text {th }}$ maximum amplitude factor with varying standard deviation from Monte Carlo simulations. The data shown in Figure 9 further confirm the conclusion that the maximum amplitude factor is very sensitive to small perturbations levels. The work from Ottarsson and Pierre also points out that moderately weak inter-blade coupling could trigger significant increases of the forced response amplitudes [45] because a too weak inter-blade coupling makes each blade acts as an isolated mistuned part that prevents the transfer of the kinetic energy between the blades. On 
the contrary, a very strong inter-blade coupling allows the energy to transfer and dissipate more rapidly, therefore lowering the possibility of mode localization. Previous work published in $[42,46,47]$ also indicates that the sensitivity of the forced response to mistuning is very much associated to the frequency-veering region shown in Figure 4. The modes in the veering region tend to feature a mixed disk-blade motion that indicates a strong inter-blade coupling, which in turns facilitates mode localization and therefore significant dynamic displacement levels when mistuning is present [45]. References [48, 49] also point out that a quantification of the veering and the determination of the corresponding nodal diameters in these veering curves can provide critical information to assess the strength of the blade-to-blade coupling, which can then be used to predict the mistuning sensitivity.

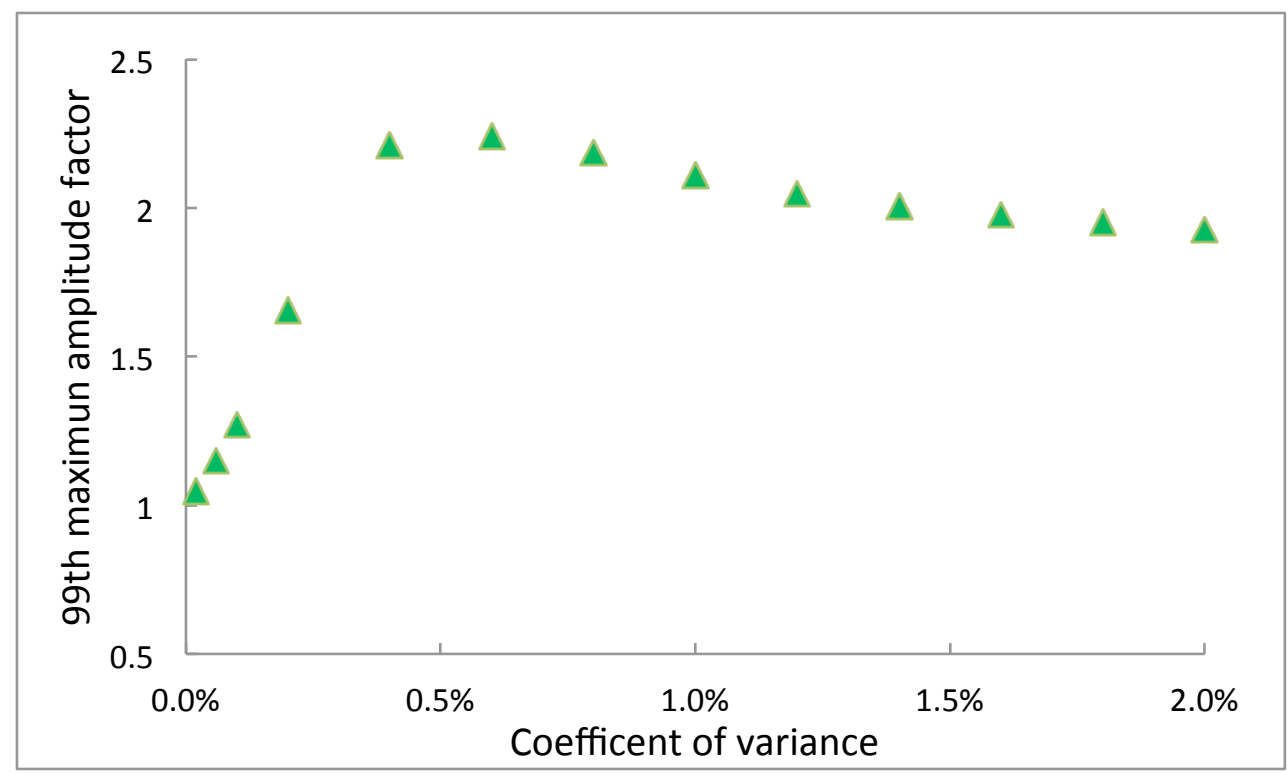

Figure $999^{\text {th }}$ max amplitude factor from Monte Carlo simulation with varying coefficient of variance [50]

\subsection{Mistuning problems in open literature}

Due to the important role of bladed disc systems in aeroengines, mistuning has generated a significant body of research within the gas turbine community for more than 50 years. It is possible to identify more than 400 papers published in the field, with the overall research broadly divided into 8 topics:

- Mechanism exploration of mistuned bladed discs

- Development of reduced order modelling techniques

- Stochastic analysis of the maximum forced response

- Mistuning identification technique \& worse blade identification (largest amplitude factor)

- Robustness design of bladed discs

- Experimental validation techniques 
A detailed literature review on mistuning before 1990 has been presented by Ewins [38]. Srinivasan has then presented an overview about bladed disk issues in [51], and further work has also been reviewed by Slater et al in 1999 [52]. A more recent review on mistuned bladed discs with a particular emphasis on the use of reduced order modeling has been presented by Castanier and Pierre [7]. Since the main focus of this research activity is about the development of reduced order prediction methods, only the relevant literature will be reviewed in the following sections. With respect to the rest of the topics listed before the reader can refer to the review papers mentioned above.

\section{Reduced order models (ROM) for rotor systems}

In this section, a review of the development of ROMs for mistuned bladed discs is provided. We firstly describe the rationale of using reduced order modeling to address mistuning, followed by a review of the literature associated to single blade ROMs. A review of different ROMs generation methods for the whole bladed discs is then presented. Finally, emerging directions to improve the fidelity of ROMs predictions are further discussed.

\subsection{Rationale}

An accurate bladed disc model not only can provide insight to the dynamics and internal mechanisms of mistuned systems but also be beneficial for the prediction of the maximum dynamic response. These estimations can be used to provide guidelines about how to adjust the design or the manufacturing process and make the system more robust. In general, analytical and numerical models of mistuning are more cost-effective than direct experimental characterizations because a large number of tests on nominally identical bladed disks are necessary to have a statistically representation of all the various sources of uncertainty leading to mistuning. High-fidelity FE models of bladed disks are therefore commonly used to predict both the maximum dynamic excitation at representative design points and the associated behavior due to pre-defined mistuning patterns $[53,54]$. Since mistuning breaks the cyclic symmetry of bladed-disk systems [7], simplified FE models of single sectors are not suitable for analysis purposes, and in most cases a full bladed disk model is necessary. A complete FE model of a bladed disk typically involves the use of millions of degrees of freedom (DOFs), making parametric analysis too expensive even on high-performance computing facilities. The potential use of probabilistic approaches (i.e., Monte Carlo simulations (MCS), improved sampling generation techniques like Latin hypercube [55]) further increases the computational costs. In terms of classical reduction techniques used in the industry, the accuracy provided by Improved Reduced System (IRS) techniques largely depends on the selection of numbers and locations of the master degree of freedoms (MDOFs) [56], as in classical Guyan reduction techniques [57]. Moreover, the latter techniques are not able to exploit the cyclic nature of the 
geometry of bladed disc systems. As a consequence, various FE-based reduced order methods (ROM) have been developed during the last two decades not only for the mistuned bladed disc but also for the blade alone.

\subsection{Single blade ROMs}

A consistent body of literature has been devoted to investigate the effects on the dynamics of the single blade generated by uncertainties associated to the geometry or material properties [58]. The blade models used in these works are mainly based on high-fidelity FE models, or coordinate measurement machine data [58-60]. State-of-the-art in ROMs to represent single rotating blades mainly consists in using simple cantilever beams with rectangular cross section to approximate the exact shape by finite element representation [61, 62]. Torsion-induced displacement are present in the vibrational patterns of these blades, and they are mainly induced by the coupling between the bending deformations in the flap wise and chord wise directions. Second order effects (like shear deformations, rotary inertia, warping of the cross section, root fixing and Coriolis accelerations) do also contribute to provide a complex dynamic displacement pattern.

The blades assembled on the compressor have been traditionally modeled using either simple twisted Euler-Bernoulli's beam approaches, or twisted Timoshenko's beam formulations with varying levels of complexity and warping [63, 64]. The dynamics of rotating pre-twisted blades with the two beam models has also been thoroughly investigated [64]. Although these simplified models are able to capture the first few fundamental modes (global bending, torsion, axial stretching and coupled bending motions in two directions), they are less suitable to represent the mode shapes of the real components. Blades are usually characterized by strongly coupled bending and torsional deformations, chord wise bending and edge wise flap. Field designers have also pointed out that the beam models provide a poor performance when they are required to predict the blade modes associated with chord wise bending, i.e. the second strip mode [63]. The uncertainty propagation analysis of these models is mainly restricted to the material properties and lumped masses at particular positions for the first few global modes [63]. Yuan et.al.[65] have also introduced an equivalent 3D frame model to describe the coupled flexural/torsional mode shapes, with their natural frequencies and associated modal masses. The frame configurations are identified through a structural identification approach based on a simulated annealing algorithm with stochastic tunneling. Figure 10 shows the comparisons of the mode shape of the 3D beam frame and the high fidelity models. The approach is considerably more accurate than state-of-the-art blade ROMs based on traditional Timoshenko beams, and provides excellent accuracy at reduced computational time when compared against high fidelity FE models. A sensitivity analysis shows that the proposed model can adequately predict the global trends of the variations of the natural frequencies when lumped masses or material properties are used for mistuning analysis. 


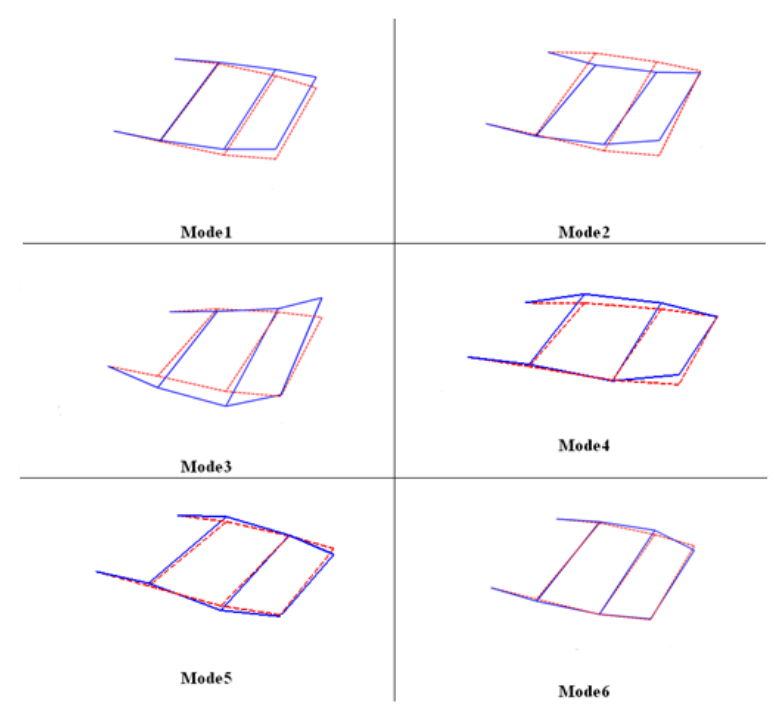

(a)

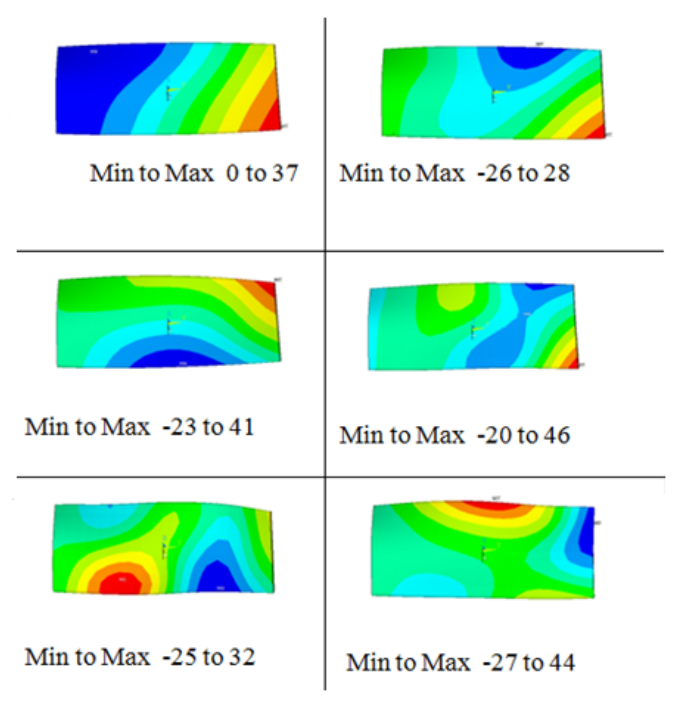

(b)

Figure 10 Comparison of (a) the mode shapes of the 3D beam frame (red line is the original shape of geometry while the blue line is mode shape) and (b) the HF models (out of plane) [65]

\subsection{Lumped parameter models}

A basic bladed disk model often used in the industry is represented by a cyclic chain of spring-mass oscillators with either one or two Degrees of Freedom (DoF) for each sector [66], namely lumped parameter model. Figure 11 shows the sketch of a 2 DOFs per sector lumped parameter model, with one DOF representing the motion of the blade and the other being related to the motion of the disk sector [67-69]. The blade DOF has stiffness $k^{b}$ and mass $m^{b}$; the parameters $k^{d}$ and $m^{d}$ represent the stiffness and mass of the disc. The stiffness $k^{c}$ is related to the coupling between blades through the disc. $q_{i}^{b}, q_{i}^{d}$ are the generalized coordinates of blade and disc in the $i^{\text {th }}$ sector respectively. These models, although simple, have been widely used at preliminary design stage since they can capture some of the fundamentals of the dynamics of bladed discs. These models also require much lower CPU times to run, compared to the computational times needed for full-scale finite element models [7]. The lumped parameter models have also shown their suitability to investigate the statistics of the forced response in mistuned bladed disks when the uncertainties are introduced into their parameters [7, 68]. However, the practical applicability of lumped parameters models is limited to the first three fundamental modes at the most [7]. 


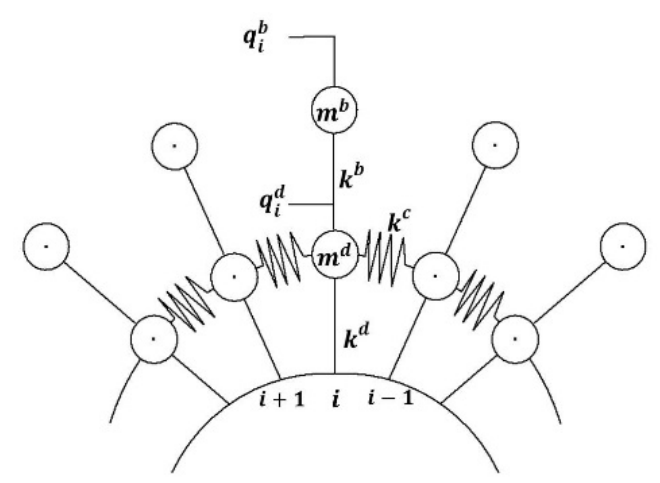

Figure 11 Two DOFs per sector model

\subsection{Beam frame assembly}

The predictive capability of mistuning phenomena provided by lumped parameter models is extended by using a beam frame assembly. Figure 12 shows a schematics of the beam frame assembly, each frame configuration being identified through a structural identification approach based on a simulated annealing algorithm [65]. The beam frames can then be assembled by using different spring-dashpot networks to represent the dynamics of the whole rotor. The beam frame assembly is able to capture the higher coupled modes than the lumped parameter model. Also, for mistuning analysis, the mass can be lumped to physical coordinates that represent the practical uncertainties from the geometry and materials. However, for better accuracy, it is preferable to take advantage of FE models to predict the dynamic response. However, due to the high computational costs involved in running high fidelity FE models, FE-based ROMs have been developed during the last two decades. These reduced order techniques allow designers to make the prediction of the dynamic response of mistuned bladed discs in an efficient manner from the computational and fidelity perspectives.

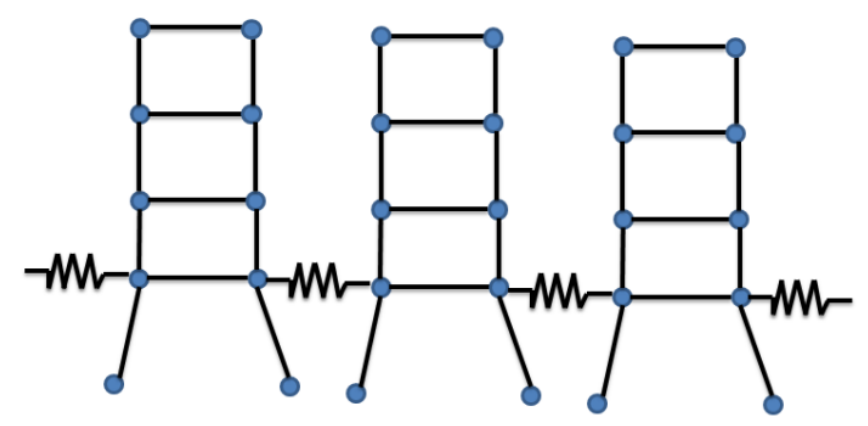

Figure 12 The assembly of the beam frame with coupling springs [70] 


\subsection{Finite element based ROMs}

\subsubsection{Component mode synthesis techniques}

The FE based ROMs used in mistuning applications can be broadly classified into two groups, both of which are based on modal reduction techniques. The first group includes techniques mainly developed between 1983 and 2000, and consists essentially in Component Mode Synthesis (CMS) methods [71-73]. CMS approaches assume that the blades and the disk are distinct sub-structural elements, therefore their modes are separately calculated via deterministic FE analyses. The modal bases of the two substructures are then employed to reduce the size of the overall system matrices by enforcing compatibility conditions at the interfaces between the blade and the disk. The compatibility between the dynamic displacements existing within the interfaces of the substructures can be described by means of fixed-interface [74], free-interface [71] and hybrid-interface methods [72]. The mistuning is then introduced into blades in a form of NF deviations. As an initial attempt, Irretier in1983 [71] applied a free-interface CMS method to FE models representing 24-bladed discs with two different scales. Compared with the results from the high fidelity FE models, they show good agreements in the free dynamic response. In 1994, a hybrid-interface CMS approach was originally proposed by Ottarsson [72]. Similar to the fix-interface approach, the blade modes are still represented with a basis of cantilevered blade modes plus a set of shapes related to the blade deformation induced by the disk. The difference in his approach lies in the fact that the disc-induced blade motions are directly included in the disc modes by using the FE analysis on the model of the disc with massless blades. Unlike the fix-interface method, this approach avoids the use of constrained modes, which further reduces the size of the ROMs. In the same period Yang \& Griffin [73] proposed a novel modeling technique able to describe the blade and disc motions at the interface by assuming the blade base motion rigid. Similarly to Ottarsson's approach, the Yang \& Griffith's technique does not require the inclusion of constraint modes. However, the two aforementioned approaches are not suitable to predict mistuning in forced vibrations because of their very simplified representation of the coupling motions. The Craig Bampton (CB) method [75] was then introduced into CMS to better capture the dynamic deformations at the blade-disc interface. However, the drawback of applying the CB approach is the significant increase of the size of the ROM. Due to this weakness, Bladh te al. [76, 77] built an extremely compact model by combining the Craig Bampton method with the cyclic disc components through the modal analysis on the intermediate CMS model.

The type of interface method affects significantly the numerical convergence of the CMS ROMs. A fixed interface can exhibit very good convergence properties by increasing the number of component modes, but it requires large numbers of interface DOFs and therefore penalizes the computational efficiency [78]. Another limitation of the fixed interface method is its inability to easily obtain the constrained modes from testing, and makes this method suitable to purely analytical or numerical models [79]. Free interface methods have a slow convergent, although in principle this problem can be overcome by using residual flexibility 
(i.e., attachment modes). These modes are obtained from using low frequency approximations to take into account the contributions from the neglected high frequency components [78]. Unfortunately, it is difficult to extract accurate residual flexibility terms from experimental data and also the information about residuals from an analytical model [79].

\subsubsection{System mode based techniques}

The second group of ROMs (System Mode based Methods - SMM)) was developed initially by Yang and Griffin in 2001 [80]. The main models belonging to this group include the Fundamental Mistuning Model (FMM) [66], the Component Mode Mistuning [81] and the Integral Mode Mistuning [35]. The main concept behind the SMM is to employ selected sets of tuned system modes as a basis to represent the tuned disk-blade system, and then to project the mistuning effect into the system as perturbations. This strategy allows in principle an exact representation of the baseline tuned system, and SMM techniques are also more computationally efficient than the CMS approaches.

The first type of the SMM includes Subset of Nominal Modes models (SNM) and FMM [66]. These models are based on the assumption that the modes of a mistuned bladed disk can be essentially represented by the linear combination of isolated blade modes in its tuned system when the mistuning is small [82]. In this way, the DOFs in mistuning bladed disk systems can be reduced to the number of clustered modes within a specific bandwidth, and the modes away from these clustered modes are neglected. The resulting errors from using the SNM technique are inversely proportional to the frequency separation between clustered and distant modes [83]. The FMM approach represents a simplified form of the SNM used when the bladed disc can be represented as an isolated family of blade-dominated modes. To assess the influence of mistuning, the only required inputs when using FMM are a set of tuned system modes and the blade-alone frequency deviations, providing a more efficient method. However, the model is not very applicable to the modes near the frequency veering region shown in Figure 2.1 and also the modes with much high modal density.

In 2002, Petrov et al [84] proposed another SMM approach that removes the restricted assumptions existing in SNM. In their approach, the response of a mistuned bladed disc is represented by a vector of forced response from a tuned system vector, and a modification matrix resulting from the mistuning. The technique makes use of the classic Sherman-Morrison-Woodbury (SMW) formula $[85,86]$ to update efficiently the forced response of a mistuned bladed disc. Using a simplified formulation, the model allows extracting the calculation of the blade response in a mistuned system and users to perform parametric studies for investigating effects of different types of uncertainties.

The considered state-of-the art in SMM techniques is the CMM developed by Lim in 2007 [81]. Lim modeled a mistuned bladed disc by using a basis of tuned normal modes and a virtual blade mistuning component. The modal and virtual bases are then assembled by using a hybrid interface method. The individual blade mistuning can be effectively projected into 
ROMs by using the modal participation factors derived from the tuned system,. In this way, no strict assumptions are introduced about the mistuning, which can be either small or large in magntidude. In 2010, Vargiu et al [35] expanded this work in an application to an integral bladed disc, in which the mistuning was introduced as a sector rather than blade mistuning. This technique has been called integral mode mistuning (IMM).

The accuracy of the SMMs is very much dependent upon the modal representation of the tuned system. It works pretty well when the technique is to present the low order mode families. However, for the high order mode families, practical industrial applications have shown that SMMs constitute reliable predictive tools that however involve the extraction of hundreds of modes from the FE full-scale models. SMM models are also not able to perform the mistuning analysis by using the direct perturbation of specific parameters in the modal domain, as they generally introduce the mistuning in the form of a NF deviation that complicates the computation of sensitivity studies required for robust design. Most importantly for sensitivity purposes is the fact that these ROMs have to be rebuilt or updated through a repeated extraction of modes from the perturbed FE models to guarantee accurate predictions.

\subsection{Emerging trends}

One of the main reasons behind some poor predictive capabilities of these two generations of ROMs is their partial intrinsic lack of capturing the physical deformation mechanisms existing in the blades or sectors. Although many important phenomena existing in rotors like damping mistuning [87, 88], stress stiffening [54], spin softening [39], Coriolis effects [89, 90] and inter-stage coupling [91-93] have been investigated and included into the ROMs, the fundamental understanding and modeling of some other physical aspects are still in development. These phenomena include the aerodynamic coupling, nonlinearities from contact friction and geometry, multistage rotor coupling and blade damage [83]. It is also worth noticing that accurate estimations of the forced dynamic response of mistuned blade systems can only be found if all the above mentioned effects are included in a model, rather than being analyzed individually.

From the industrial perspective, FE-based ROMs can effectively reduce the computational effort required by the high fidelity models. As these ROMs are all based on modal reduction techniques, it is necessary to obtain the modal information from the tuned system before their implementation in the mistuned system. In order to accurately predict the dynamic response of a mistuned system in the high modes, several hundreds of modes from a full-scale FE model as the basis are usually necessary $[94,95]$. At industrial design level it is however still very time consuming to make use of these models for assessing the impact of different mistuning patterns on the dynamic behavior of a bladed disc. Although the lumped parameter approach can be used, this simple model can only provide a fundamental understanding of the mistuned bladed disc for the first few modes and it is not suitable to capture higher coupled modes in a large frequency range. Therefore, there is still a demand within the gas turbine industry to develop simple design tools for the mistuning analysis that can provide higher 
accuracy compared to the one from a lumped parameter model, and be less computationally expensive than a FE based model.

\section{Stochastic techniques for mistuning problems}

In this section we will review previous efforts performed about the stochastics analysis in mistuned bladed disc systems. The rationale of the investigation is firstly described, followed by a review of the current stochastic techniques for reliability analysis, especially on Subset Simulation that has the great potential to improve the computational efficiency of statistical estimations performed on mistuned bladed discs.

\subsection{Rationale}

As discussed before the blade properties in a bladed disc assembly always vary in a stochastic manner due to uncertainties from the manufacturing process, in-service wear and lack of material homogeneity. The results from the presence of uncertainties in the blades may compromise the high-cycle fatigue resistance of bladed disks, reducing the endurance and the reliability of the whole engine. It is therefore essential to assess the effects of these uncertainties on the maximum dynamic response in any of the blades of the assembly. Luckily, the high-percentile blade forced response obtained from the stochastic analysis can be effectively used as an indicator to quantify the influence of such uncertainties on the fatigue life, and also reasonably estimate the high cycle fatigue endurance [68]. Methods for stochastic analysis include Monte Carlo simulation (MCS), reliability methods, perturbation methods, spectral approaches and stochastic reduced basis techniques [68, 96-98]. During the analysis, the uncertainty is commonly introduced by using added masses, spring elements or deviations from the nominal material properties of the blades. However, the reliability analysis for mistuned bladed discs usually suffers from high computational costs. High-fidelity FE models of bladed disks are commonly used to predict the maximum dynamic response at representative design points [53, 54]. Unfortunately, a complete FE model of a bladed disk typically includes millions of DOFs, making the stochastic analysis too expensive even when using state-of-the-art high-performance computing facilities [99]. The problem becomes even more intractable by the fact that simplified FE models of single sectors cannot be used, since mistuning breaks the cyclic symmetry of bladed disc systems.

\subsection{Current stochastic methods used in mistuning}

\subsubsection{Monte Carlo method}

MCS are commonly used in stochastic analysis of mistuned bladed disc systems through the use of repeated deterministic experiments. The popularity of the MCS is due to their highly 
computational robustness with respect to the dimension and the performance function of the problem [100]. The main drawback of the standard MCS is represented by the fact that it requires a large number of samples to ensure the representation of the entire parameter range. The convergence rate of the MCS is in the order of $1 / \sqrt{N}$ (where $N$ is the sample population) [98]. For the components in a gas turbine engine, the allowance of failure probability tends to be extremely small (commonly under $10^{-3}$ ) under the given number of operational cycles $[7,68,101,102]$. For a full-scale structure with a large number of degrees of freedom (DOFs), the computational costs increase significantly, sometimes reaching unacceptable levels. As a result, parallel computation and processing is used to accelerate the convergence [103], as well as Accelerated MCS and advanced sampling techniques (which will be introduced in the following sections). Another problem of using the MCS is that there is not a clear rule about how to determine the sample number required for a particular problem [104]. In most cases, extremely large values of $\mathrm{N}$ have to be selected to ensure convergence, however this may lead to severe computational penalties. To quantify the convergence properties of direct MCS methods, Yuan et.al introduce the concept of the confidence interval [50]. The case study confirms that it can be effectively used in the MCS.

\subsubsection{Accelerated Monte Carlo method}

Accelerated MCS has also been widely used in mistuning analysis, its computational advantage being fit to an assumed probabilistic distribution with few realizations from MCS [83]. Although efficient, much debate has focused on the suitable type of distribution to represent the maximum blade forced response, being either a Weibull [105-107] or a Gaussian function [108]. Sanliturk et al have concluded that there is no unique distribution to describe blade amplitudes under different engine orders of excitation, and that accelerated MCS is therefore not a general approach to predict the statistics in mistuning problems [109].

\subsubsection{Sampling techniques}

Various methods have also been developed for efficient sampling from the input parameter probability function. Unlike the standard MSC using pseudorandom sequence, Quasi-Monte Carlo methods sample the input variables using low-discrepancy sequences, with typical examples of Halton and Sobol sequences [110, 111]. That quasi-random sequence can improve the convergence rate to the order of $1 / N$. The structured sampling techniques, such as stratified sampling and Latin hypercube sampling, can be used to further improve the computational efficacy. In stratified sampling, the sample space is participated into a number of strata or subgroups, with each stratum having a specified probability of occurrence. Random samples are then proportionally selected from the each stratum [112]. Latin Hypercube Sampling technique can ensure the dense coverage of the range of each input variables without the demanding definition of the strata and the related calculation of their probabilities. The rationale behind it is to divide each of $\iota$ input parameter's range into disjoint $\Omega$ intervals of equal probability and randomly generate one sample in each corresponding interval. The samples of each variable are then randomly matched together until obtaining $\Omega$ l-tuples $[113,114]$. 
The other way to improve the convergence rate consists in the optimization of the location of the samples for MCS, which is known as variance reduction techniques. Importance Sampling (IS) is a typical example of the variance reduction techniques. The rationale of this method consists in shifting the underlying distribution towards the failure region to gain information about rare events [115]. The success of IS very much depends on a careful choice of the importance sampling density distribution, which requires a prior knowledge of the system in the failure region. The method works extremely well when applied to relatively low-dimensional static and dynamic problems because when the dimension of the uncertain parameter space is not too large the failure region is relatively simple to describe. However, for structural problems with large model size IS is generally shown to have a poor efficiency, as demonstrated by Lee [68] using a radius-based IS method [116] for mistuning analysis due to the large number of stochastic variables that influence the mistuning behavior, and the associated lack of information about their a priori influence on the system failure region. Chan [104] recently proposed a new procedure to overcome the lack of such background knowledge for mistuning problems by firstly using an optimization analysis to identify the failure region and then employing IS to characterize the corresponding conditional probabilities. The method is able to estimate very small conditional probabilities, but the identification step of the failure region is still computationally demanding.

\subsubsection{Analytical methods}

Analytical methods are also available to evaluate the failure probability of a system containing uncertainties, such as traditional points-based methods (including both first and second order reliability methods) and mean value procedures [117]. In the field of the reliability analysis, first order reliability method is one of the most widely used approximation techniques. The method simulates the limit state surface (defined failure state) to a hyperplane and neglects the higher orders of expansion series. The reliability index is defined as the smallest distance from the origin to a point on the approximated hyperplane in a standardized normal space. The accuracy of the method depends obviously about how much the hyperplane is close to the real surface of the limit state and far from the original point. Therefore, if the surface is represented by a complicated geometry and close to the origin, the statistics estimated by first order reliability method may be not very reliable. To improve the accuracy of the first order method, second order reliability methods has been also developed [118]. Second order methods make use of a quadratic surface to approximate a limit state surface around the most probable points in a standardized normal space, and are able to better represent the limit state surface. Second order methods have been extensively applied to structural safety problems [119]. In open literature is also mentioned the mean value first order reliability method based on a first-order Tylor series approximation of the performance function that linearized at the mean values of the random variables. The advanced mean value method is another further development proposed to improve the approximation of the mean value first order method [120]. The principle behind this technique is essentially to employ the most probable points of the first order approximated limit state surface and compensate for the truncation error in order to reduce the error in probability estimate. In the past, these 
low-order reliability methods have been investigated for mistuning applications [68] and found generally to fail the prediction of the distribution of the maximum amplitudes with the stochastic variables, because of the complicated limit state surface function commonly used to define mistuning problems [68]. The number of dimension in this reliability problem is quite high, which at least equals to the number of blades in a rotor. The high dimension in mistuning problem also makes difficult to provide accurate estimations of the statistics in the highly nonlinear performance functions typical of mistuning.

With respect to the stochastic FE methods, the perturbation method is one of the most popular analytic techniques used for analyzing systems in stochastic engineering [98]. The method approximates the solution of the perturbed system by a Taylor series expansion around the mean value of the random variables. The mean and covariance can be then simply expressed in terms of the expansion coefficients and the statistics of the random variable. In the stochastic analysis of the system represented by FE model, the method is very computational efficient because the matrix inversion is only required once for evaluating the nominal solution of the system. However, due to the limitations in numerical differentiation, the expansion terms up to the second order is commonly used, and thus only the first and second order statistics are computed. As a result, this analytical method is only accurate in the case of the very small uncertainties [121]. More recent approaches to stochastic simulation of systems subject to uncertainty include the polynomial chaos (PC) approach and based on Wiener's theory of homogeneous chaos [122]. The stochastic process is represented through a spectral expansion using orthogonal polynomials. The optimal convergence is usually obtained for Gaussian stochastic process. However, recent extensions to this method shows the optimal convergence can be achieved for more general stochastic phenomenon, with the use of orthogonal polynomial basis functions of Askey scheme to approximate the random processes [122]. Polynomial chaos has been popular in applications related to fluid and structural mechanics. Sinha in 2003 [123] has developed a methodology to apply this technique to mistuning problems. Compared with the MCS, PC allows to compute efficiently the statistical response of the mistuned bladed disc. However, the estimation of a large number of the expansion coefficients is rather cumbersome and time-consuming.

\subsubsection{Surface response methods}

Various response methods have been developed to address the response of a system with limited number of data. Most of the methods are known as global approaches, and tend to use a single quadratic or cubic polynomial to represent the uncertainty space. Therefore, these techniques may introduce large errors when trying to approximate a nonlinear performance function such as in mistuning applications. The state-of-the-art method that best describes the locally nonlinear behavior of the response consists in using a moving least squares response surface construction, which is widely adopted in meshless methods [124]. The method consists in a series of compact supported weighting functions and it is capable to approximate the highly nonlinear response surfaces in mistuning problems. However, the accuracy of the response surface commonly depends on the number and location of the sampling points. Sinha [108] has also employed artificial neural networks (ANN) for the statistical characterization of the forced response as a 
function of the input parameters. These methods however usually require a very large number of sampling points for the training, which makes extremely difficult their usage in a large-scale mistuning system [125].

\subsubsection{Subset Simulations}

$\mathrm{Au}$ et.al [126] proposed the SubSim technique in engineering reliability problems to efficiently compute small failure probabilities. The basic idea behind SubSim is to express the failure probability as a product of larger conditional failures by introducing intermediate failure events. With a proper choice of these intermediate events, the conditional failure probabilities can be made sufficiently large and can therefore be estimated by means of simulations with a small number of samples. The original version of SubSim makes use of the Markov chain Monte Carlo (MCMC) [127] technique, based on the modified Metropolis-Hastings algorithm for generating conditional samples [128, 129]. Two newer variants to generate conditional samples have recently been developed for the causality exploration of dynamical systems. SubSim/Splitting is the first variant that is applicable to first-passage problems involving deterministic dynamical systems subjected to stochastic excitation and makes use of trajectory splitting for generating conditional samples [130]. Another variant called SubSim/Hybrid uses a combined MCMC/Splitting strategy and so has the advantages of both MCMC and splitting; it is applicable to both uncertain and deterministic dynamical systems. These newer versions of SubSim use the same adaptive procedure of the original technique to generate conditional samples for higher levels based on those from lower levels. The performance of these three SubSim methods have been compared in Ref [131]. All three SubSim variants are proved to be effective in treating problems with large numbers of stochastic values. The two variants using splitting are not however considerably superior to the original version.

Yuan et al [50] assess of the performance of using SubSim techniques to increase the computational efficiency for the prediction of the probabilistic dynamic behavior in mistuned bladed disc systems. The technique is shown to capture efficiently the statistical properties of the mistuned blades with less than 5\% samples compared to the direct MCS method. As discussed in Ref [126], the advantage of adopting SubSim techniques consists in its robustness with respect to the dimension and its efficiency to estimate small probabilities. Most importantly, no prior knowledge of the system is required, which makes this method a strong candidate for the stochastic analysis of mistuned bladed disc systems.

\section{Efficient matrix inversion methods for the stochastic analysis}

In this last section, some efficient stochastic techniques to predict the probabilistic forced response dynamics of a mistuned bladed disc have been reviewed. In this section, an emphasis of the review is on the efficient matrix inversion techniques for stochastic analysis of the system represented by FE model, in particular for what it concerns Neumann expansion methods and the Sherman Morrison formula. 


\subsection{Rationale}

The maximum amplitude factor of a mistuned bladed disc is usually represented within a non-convex performance function with stochastic inputs, and it is usually referred to the deviational coefficients of the blade stiffness or NFs [132]. The dimension of this reliability problem is as large as the number of blades assembled in the rotor. The high dimension and complex failure region of the mistuning problem makes the use of analytical methods generally inefficient for reliability analysis [126]. These methods are always unable to provide satisfactory results in a computationally efficient way. Simulation methods provide viable means to compute small failure probability of the mistuning problem. However, even with the use of the advanced sampling or SubSim techniques, they stiff suffer from the intrinsic high computational costs, because thousands of samples are often necessary to provide reliable statistics estimates. Bladed disks in turbomachinery rotors are commonly represented using detailed FE models with dimensions well in excess of millions of DOFs. The high computational costs of the mistuning analysis are essentially related to the repeated inversions of the dynamic matrix for either full scale model or any type of ROMs. Also, the matrix after the inversion will not be banded anymore, and thus the following matrix multiplication cannot be performed efficiently. Efficient inversion techniques are therefore highly sought after, not only because thousands of simulations are required in stochastic methods, but also because the size of the dynamic matrix in bladed disc systems is often large.

\subsection{Current methods}

\subsubsection{Matrix factorization methods}

Matrix factorization methods are commonly used to reduce the computational costs associated with matrix inversions. The main idea behind these techniques is to express the dynamic stiffness as a product between two banded triangular matrices, consisting of permutations of lower and upper triangular matrixes. In this way, the inversion of the large matrix can be efficiently computed by inverting two banded sub-matrices. In the case of the static stiffness matrix the Cholesky decomposition is commonly applied to obtain the lower triangular matrix [133]. However, Cholesky decomposition is not suitable for dynamic stiffness calculations, as the existence of positive and real diagonal elements in the matrix is no longer guaranteed due to the added damping matrix. LU matrix factorization techniques are therefore commonly used to reduce the computational costs in inversions of dynamic FEMs. LU expresses the dynamic stiffness as a product between two essentially triangular matrices, including a permutation of a lower triangular and an upper triangular matrix [134].

\subsubsection{Sherman-Morrison-Woodbury formula}

The repeated inversions in the stochastic analysis can also be potentially avoided by using the Sherman-Morrison-Woodbury (SMW) formula. The SMW formula gives an explicit and 
efficient expression for the inverse of a perturbed matrix by adding a rank-one update based on the knowledge of the unperturbed inverse matrix (also called the 'Matrix Inversion Lemma') [135]. The main advantage of using the matrix identity formula is to eliminate expensive repeated inversions in the stochastic analysis, therefore obtaining a significant reduction of the associated computational costs. Due to this fact, the SMW formula has been applied to the dynamic response of a simple linear system in [136], and also successfully used to calculate variations in the non-linear analysis of systems with friction dampers [137]. Moreover, the method enables the mistuning analysis of bladed discs using large FEMs. The knowledge of the unperturbed system is obtained from the analysis of tuned bladed discs based on cyclic symmetry approach [138]. Recently, Yuan.et.al [139] made an assessment about using the SMW formula and the Neumann expansion method, to enhance the computational efficiency of the stochastic analysis in mistuned bladed disc systems. The modified SMW algorithm can be efficiently used to update the inversion of an uncertain matrix without the need of multiple separated inversions. The computing time can be reduced up to $20 \%$ compared with the LU factorization approach. The associated computational advantage however depends on the ratio of the number of the mistuned blades introduced with respect to the size of the representative model. The method becomes inefficient and even more expensive when more than two blades are perturbed in the current model. However, validations of the approach proposed here are required for high large-scale FE models.

\subsubsection{Neumann Expansion method}

The Neumann Expansion Method (NEM) provides an alternative approach to circumvent the direct inversion of an uncertain dynamic stiffness matrix [121]. The NEM technique represents the uncertainty matrix as the sum of deterministic and stochastic matrixes. Neumann series are then used to expand the coefficients of the perturbation matrix, and the solution of the inversion problem is represented as a series. The technique is based on an iterative process involving the solution of hierarchical linear algebraic equations. The fluctuation range permitted by the NEM is generally higher than the one allowed in other perturbation techniques, because it is easier to incorporate high order terms [140]. However, NEM is often considered not suitable for problems with large random variations, because the spectral radius of the iterative matrix must be smaller than one [141, 142]. A scalar-modified NEM approach has been proposed to guarantee an effective convergence under large perturbations [121]. The computational costs of the Neumann expansion method generally depend on the number of terms required in the resulting series. To achieve a high level of accuracy, this new NEM techniques however requires more terms in the series, which may lead to higher computational costs even compared to a direct matrix inversion. For the application in mistuning problem. Reference [139] shows NEM can decrease the computational costs by $200 \%$ compared with the matrix factorization approaches, with a maximum relative error lower than $2 \%$. The convergence of the Neumann expansion is however not guaranteed when the excitation frequency approaches resonance. The convergence of the Neumann expansion method improves at a high damping and low mistuning levels. From a practical point of view, both damping and mistuning levels are in general pre-determined and cannot be easily altered. The scalar-modified Neumann expansion 
can reduce the extent of the divergence region near resonance by $50 \%$, and eliminate "tout court" the divergence at low perturbation levels. However, its convergence cannot be completely ensured within the range of damping and stiffness perturbation used in the current reduced order model. In order to overcome the divergent problem, it would be interesting to employ a hybrid techniques based on the use of stochastic Neumann expansion in the frequency domain, coupled with a matrix factorization in the neighborhood of the resonant frequencies. In this way, the advantage of the NEM can be retained during the dynamic analysis.

\section{Summary}

In this paper, a general introduction to basic definitions, terminology and dynamic characteristics of bladed discs systems are given. Previous studies about vibration problems and related suppression techniques in gas turbine engines bladed discs are reviewed. We have also looked at the origins of the mistuning phenomenon in bladed discs and their effect on the reliability and durability of a jet engine.

One of the main focuses of this paper has been about the review of ROM approaches for mistuned bladed discs and single fan blades developed within the last two decades. The lumped parameter model is still widely used at the preliminary design stage for mistuning. However, its practical application is limited at best to the first three fundamental modes. Although still more efficient than their full-scale model counterpart, state-of-the-art FE based ROMs are limited in some ways for industrial applications, mainly because it is necessary to extract a large number of the modes from a high fidelity model in order to guarantee the given accuracy when high order mode families are of most interests. The time associated to the preparation and the extraction of the modal basis in these techniques is quite large and time-consuming for preliminary design stages with high fidelity. Moreover, the intrinsic nature of the CMS or SMM approaches makes difficult the execution of parametric studies for robustness design. For single blade ROMs, state of the art models such as Timonshenko beam or twisted beam are also limited because they can only represent the first few fundamental modes. These ROMs are less suitable to describe the coupled bending and torsional motions, let alone the edge wise mode of fan blades. However, beam frame model is more capable to capture the higher/couple model with limited increase of computational cost.

The review also focuses on the use of efficient simulation and inversion techniques to obtain the probabilistic behaviour of the mistuned bladed discs. Reliable statistics estimates of the maximum amplitude factor are critically important to qualify the effect of the mistuning phenomenon on the fatigue life of the bladed disc. Analytical techniques are in general inadequate to perform a consistent reliability analysis of a mistuned bladed disc due to the high dimension and complex failure region of the mistuned system. Simulation-based methods such as MCS, IS and accelerated MCS provide a feasible approach to estimate the failure probability of this problem. However, all these methods are limited in different ways because large numbers of samples are required, presence of unknown failure regions, or the 
proposed distribution functions are unsuitable. We have also reviewed novel simulation techniques applied to mistuning problems and other engineering applications. The SubSim technique is the one of the most interesting currently available, and the properties of this approach make this method a strong candidate for the stochastic analysis of mistuned bladed disc systems.

We have also investigated inversion techniques to increase the computational efficiency of the forced response stochastic analysis of the mistuning system. Essentially, the high computational costs of simulation-based approaches are mainly due to the repeated inversions of the dynamic matrix. Given the review of the state of art inversion techniques, there is the possibility to significantly reduce the computational efforts. One of the effective ways to deal with high-scale dynamic stiffness matrices is using the LU matrix factorization method. A review of SMW and NEM methods have been also provided. These techniques show some potential to increase the computational efficiency of simulation based methods. The SMW algorithm can be efficiently used to update the inversion of a matrix with uncertainties quantifications, but its computational efficiency is limited when large number of uncertainties is introduced. NEM techniques can significantly decrease the computational costs with a maximum relative error lower than $2 \%$. However, its convergence cannot be completely ensured even with the use of scalar-modified Neumann expansion techniques.

\section{Acknowledgement}

The authors would like to acknowledge the support of Rolls-Royce plc for the support of this research through the Composites University Technology Centre (UTC) at the University of Bristol, UK. Special acknowledgement goes also to the Strategic Investment in Low carbon Engine Technology (SILOET) programme supported by Rolls-Royce plc and Technology Strategy Board (TSB), and to the China Scholarship Council. The authors would also like to thank the Reviewers for their useful suggestions.

\section{Reference}

1. Kumar, K., D.A. Prasad, and D.K. Ramchandra, Critical issues in assessment of over speed and burst margin in aero engine discs. International Journal of Computer Applications in Engineering Technology and Sciences, 2010. 2(1): p. 85-90.

2. Klauke, T., U. Strehlau, and A. Kühhorn, Integer frequency veering of mistuned blade integrated disks. Journal of Turbomachinery, 2013. 135(6): p. 061004.

3. Castanier, M.P. and C. Pierre, Consideration on the benefits of intentional blade mistuning for the forced response of turbomachinery rotors. Analysis and design issues for modern aerospace vehicles- 1997, 1997: p. 419-425.

4. Sever, I.A., Experimental validation of turbomachinery blade vibration predictions. 2004, Imperial College London (University of London).

5. Zhan, H.-j., W.-s. Zhao, and G. Wang, Manufacturing turbine blisks. Aircraft Engineering and 
Aerospace Technology, 2000. 72(3): p. 247-252.

6. Thomas, D.L., Dynamics of rotationally periodic structures. International Journal for Numerical Methods in Engineering, 1979. 14(1): p. 81-102.

7. Castanier, M.P. and C. Pierre, Modeling and Analysis of Mistuned Bladed Disk Vibration: Current Status and Emerging Directions. Journal of Propulsion and Power, 2006. 22(2): p. 384-396.

8. Kenyon, J., J. Griffin, and N. Kim, Frequency veering effects on mistuned bladed disk forced response. Journal of propulsion and power, 2004. 20(5): p. 863-870.

9. Heckel, T., A.G. Tovar, and H.-J. Christ, Fatigue of the near-alpha Ti-alloy Ti6242. Experimental mechanics, 2010. 50(4): p. 483-489.

10. Ewins, D.J., Control of vibration and resonance in aero engines and rotating machinery - An overview. International Journal of Pressure Vessels and Piping, 2010. 87(9): p. 504-510.

11. El-Bayoumy, L. and A. Srinivasan, Influence of mistuning on rotor-blade vibrations. AIAA Journal, 1975. 13(4): p. 460-464.

12. Campus, S.K., Effects of damping and varying contact area at blade-disk joints in forced response analysis of bladed disk assemblies. Journal of turbomachinery, 2006. 128: p. 403.

13. Gowda, B.M.A., H.R. Yeshovanth, and C. Siddaraju, Investigation and Efficient Modeling of an Dovetail Attachment in Aero-engine. Procedia Materials Science, 2014. 5: p. 1873-1879.

14. Sinclair, G.B. and N.G. Cormier, Contact Stresses in Dovetail Attachments: Physical Modeling. Journal of Engineering for Gas Turbines and Power, 2002. 124(2): p. 325.

15. Mikolajczak, A., et al., Advances in fan and compressor blade flutter analysis and predictions. Journal of Aircraft, 1975. 12(4): p. 325-332.

16. Rajasekaran, R. and D. Nowell, Fretting fatigue in dovetail blade roots: Experiment and analysis. Tribology International, 2006. 39(10): p. 1277-1285.

17. Gallego-Garrido, J., et al., A Class of Methods for the Analysis of Blade Tip Timing Data from Bladed Assemblies Undergoing Simultaneous Resonances-Part II: Experimental Validation. International Journal of Rotating Machinery, 2007. 2007.

18. Gallego-Garrido, J., G. Dimitriadis, and J.R. Wright, A class of methods for the analysis of blade tip timing data from bladed assemblies undergoing simultaneous resonances-Part I: Theoretical development. International Journal of Rotating Machinery, 2007. 2007.

19. Procházka, P. and F. Vaněk, Non-contact systems for monitoring blade vibrations of steam turbines. ISMA-USD conference, Leuven, Belgium, 2012.

20. Strother, O.M., Component with internal damping. 2008, Google Patents.

21. Lira, C., F. Scarpa, and R. Rajasekaran, A Gradient Cellular Core for Aeroengine Fan Blades Based on Auxetic Configurations. Journal of Intelligent Material Systems and Structures, 2011. 22(9): p. 907-917.

22. Srinivasan, A. and D. Cutts. Dry friction damping mechanisms in engine blades. in ASME 1982 International Gas Turbine Conference and Exhibit. 1982. American Society of Mechanical Engineers.

23. Johnson, C.D. and D.A. Kienholz, Finite element prediction of damping in structures with constrained viscoelastic layers. AIAA Journal, 1982. 20(9): p. 1284-1290.

24. Rhys-Jones, T., Coatings for blade and vane applications in gas turbines. Corrosion Science, 1989. 29(6): p. 623-646.

25. Sladojevic, I., et al. Forced response variation of aerodynamically and structurally mistuned 
turbo-machinery rotors. in ASME Turbo Expo 2006: Power for Land, Sea, and Air. 2006. American Society of Mechanical Engineers.

26. Mansfield, E. and A. Sobey, Fiber Composite Helicopter Blade. 1. Stiffness Properties. 2. Prospects for Aeroelastic Tailoring. Aeronautical Quarterly, 1979. 30(MAY): p. 413-449.

27. Smith, E., Vibration and flutter of stiff-inplane elastically tailored composite rotor blades. Mathematical and computer modelling, 1994. 19(3): p. 27-45.

28. De Queiroz, M.S. and D.M. Dawson, Nonlinear control of active magnetic bearings: $a$ backstepping approach. IEEE Transactions on Control Systems Technology, 1996. 4(5): p. 545-552.

29. Ewins, D. and Z. Han, Resonant vibration levels of a mistuned bladed disk. Journal of Vibration Acoustics Stress and Reliability in Design, 1984. 106: p. 211.

30. Armstrong, E.K., An investigation into the coupling between turbine disc and blade vibrations. 1955, University of Cambridge.

31. Tobias, S.A. and R.N. Arnold, The Influence of Dynamical Imperfection on the Vibration of Rotating Disks. Proceedings of the Institution of Mechanical Engineers, 1957. 171(1): p. 669-690.

32. Whitehead, D.S., Effect of Mistuning on the Vibration of Turbo-Machine Blades Induced by Wakes. Journal of Mechanical Engineering Science, 1966. 8(1): p. 15-21.

33. Ewins, D.J., The effects of detuning upon the forced vibrations of bladed disks. Journal of Sound and Vibration, 1969. 9(1): p. 65-79.

34. Dye, R.C.F., T.A. Henry, and E. American Society of Mechanical, Vibration amplitudes of compressor blades resulting from scatter in blade natural frequencies. 1968, New York, N.Y.: ASME.

35. Vargiu, P., et al., A reduced order model based on sector mistuning for the dynamic analysis of mistuned bladed disks. International Journal of Mechanical Sciences, 2011. 53(8): p. 639-646.

36. Chan, Y.J. and D.J. Ewins, Management of the variability of vibration response levels in mistuned bladed discs using robust design concepts. Part 1. Mechanical Systems and Signal Processing, 2010. 24(8): p. 2777-2791.

37. Lim, S.H.C., M.P; Pierre,C; , Predicting mistuned blade amplitude bounds and stress increases from energy formulations, in 9th National Turbine Engine HCF Conference. 2004.

38. Ewins, D.J., The Effects of Blade Mistuning on Vibration Response- A Survey, in IFToMM 4th International Conference on Rotordynamics, Prague, Czechoslovakia. 1991.

39. Petrov, E. and D. Ewins, Analysis of the worst mistuning patterns in bladed disk assemblies. Journal of turbomachinery, 2003. 125(4): p. 623-631.

40. Hodges, C.H., Confinement of vibration by structural irregularity. Journal of Sound and Vibration, 1982. 82(3): p. 411-424.

41. Hodges, C. and J. Woodhouse, Vibration isolation from irregularity in a nearly periodic structure: Theory and measurements. The Journal of the Acoustical Society of America, 1983. 74: p. 894.

42. Pierre, C., Mode localization and eigenvalue loci veering phenomena in disordered structures. Journal of Sound and Vibration, 1988. 126(3): p. 485-502.

43. MacBain, J. and P. Whaley, Maximum resonant response of mistuned bladed disks. Journal of Vibration Acoustics Stress and Reliability in Design, 1984. 106: p. 218. 
44. Whitehead, D., The maximum factor by which forced vibration of blades can increase due to mistuning. Journal of Engineering for Gas turbines and Power, 1998. 120(1): p. 115-119.

45. Ottarsson, G.P., C, On the Effects of Interblade Coupling on the Statistics ofMaximumForced Response Amplitudes in Mistuned Bladed Disks, in Proceedings of the 36th AIAA/ASME/ASCE/AHS/ASC Structures,Structural Dynamics and Materials Conference. 1995, AIAA: New York. p. 3070-3078.

46. Gottlieb, H.P.W., Extension of a text-book problem to curve veering for coupled pendulums. Journal of Sound and Vibration, 1987. 113(1): p. 185-187.

47. Jei, Y.-G. and C.-W. Lee, Does Curve Veering Occur in the Eigenvalue Problem of Rotors? J Vib Acoust, 1992. 114(1): p. 32-36.

48. Hussein, M.I., C. Pierre, and M.P. Castanier, Correlation of tuned free vibration characteristics with mistuning sensitivity for a bladed disk. Ann Arbor, 2003. 1001: p. 48109-2125.

49. Bladh, R., et al., Dynamic response predictions for a mistuned industrial turbomachinery rotor using reduced-order modeling. Journal of Engineering for Gas turbines and Power, 2002. 124(2): p. 311-324.

50. Yuan, J., et al., Probabilistic dynamics of mistuned bladed disc systems using subset simulation. Journal of Sound and Vibration, 2015. 350: p. 185-198.

51. Srinivasan, A.V., Flutter and resonant vibration characteristics of engine blades. Transactions of the ASME. Journal of Engineering for Gas Turbines and Power, 1997. 119(4): p. 742-775.

52. Slater, J.C., G.R. Minkiewicz, and A.J. Blair, Forced response of bladed disk assemblies-a survey. Shock and Vibration Digest, 1999. 31(1): p. 17-24.

53. Imregun, M. and D. Ewins. Aeroelastic vibration analysis of tuned and mistuned bladed systems. in Proceedings of the Second Symposium on Unsteady Aerodynamics of Turbomachines and Propellers, Cambridge, UK. 1984.

54. Rzadkowski, R., The general model of free vibrations of mistuned bladed discs, part $i$ : Theory. Journal of Sound and Vibration, 1994. 173(3): p. 377-393.

55. Stein, M., Large sample properties of simulations using Latin hypercube sampling. Technometrics, 1987. 29(2): p. 143-151.

56. Al-Aghbari, M., F. Scarpa, and W. Staszewski, On the orthogonal wavelet transform for model reduction/synthesis of structures. Journal of Sound Vibration, 2002. 254: p. 805-817.

57. Friswell, M., S. Garvey, and J. Penny, Model reduction using dynamic and iterated IRS techniques. Journal of sound and vibration, 1995. 186(2): p. 311-323.

58. Sinha, A., et al., Vibratory Parameters of Blades From Coordinate Measurement Machine Data. Journal of Turbomachinery, 2008. 130(1): p. 011013.

59. Brown, J.M., J. Slater, and R.V. Grandhi. Probabilistic analysis of geometric uncertainty effects on blade modal response. in ASME Turbo Expo 2003, collocated with the 2003 International Joint Power Generation Conference. 2003. American Society of Mechanical Engineers.

60. Garzon, V.E. and D.L. Darmofal. Impact of geometric variability on axial compressor performance. in ASME Turbo Expo 2003, collocated with the 2003 International Joint Power Generation Conference. 2003. American Society of Mechanical Engineers.

61. Malcolm, D.J. and D.L. Laird. Modeling of blades as equivalent beams for aeroelastic analysis. in 2003 ASME Wind Energy Symposium AIAA/ASME. 2003.

62. Hach, A.M.T., et al., Aeroelastic Analysis of a Turbine Blade Using a Reduced-order Model. 2007: Carleton University (Canada). 
63. Sinha, S.K. and K.E. Turner, Natural frequencies of a pre-twisted blade in a centrifugal force field. Journal of Sound and Vibration, 2011. 330(11): p. 2655-2681.

64. Zhu, T.L., The vibrations of pre-twisted rotating Timoshenko beams by the Rayleigh-Ritz method. Computational Mechanics, 2010. 47(4): p. 395-408.

65. Yuan, J., et al., Novel parametric reduced order model for aeroengine blade dynamics. Mechanical Systems and Signal Processing, 2015. 62: p. 235-253.

66. Feiner, D.M. and J. Griffin, A fundamental model of mistuning for a single family of modes. Journal of turbomachinery, 2002. 124(4): p. 597-605.

67. Griffin, J. and T. Hoosac, Model development and statistical investigation of turbine blade mistuning. Journal of Vibration Acoustics Stress and Reliability in Design, 1984. 106: p. 204.

68. Lee, S.-Y., M.P. Castanier, and C. Pierre. Assessment of probabilistic methods for mistuned bladed disk vibration. in Proceedings of the 46th AIAA/ASME/ASCE/AHS/ASC Structures, Structural dynamics, and Materials Conference, AIAA-2005-1990. 2005.

69. Yuan, J., et al., numerical assessment of using Sherman-Morrison, Neumann expansion techniques for stochastic analysis of mistuned bladed disc systems, in Proceedings of ASME Turbo Expo 2015: Turbine Technical Conference and Exposition. 2015, ASME: Montréal, Canada

70. Yuan, J., Scarpa, F, Titurus, B, Allegri, G, Patsias, S \& Rajasekaran, R, Novel frame model for mistuning analysis of bladed disc systems. Journal of Vibrations and Acoustics, 2016.

71. Irretier, H., ed. Spectral Analysis of Mistuned Bladed Disk Assemblies by Component Mode Synthesis. Vibrations of Bladed Disk Assemblies, ed. D.J.E.A.V. Srinivasan. 1983, American Society of Mechanical Engineers: New York.

72. Óttarsson, G., Dynamic modeling and vibration analysis of mistuned bladed disks. 1994, University of Michigan.

73. Yang, M.-T. and J. Griffin, A reduced order approach for the vibration of mistuned bladed disk assemblies. Journal of Engineering for Gas turbines and Power, 1997. 119(1): p. 161-167.

74. Joo, W., et al., Dynamic analysis of structural systems using component modes. AIAA journal, 1965. 3(4): p. 678-685.

75. Durham, W.C., M. Bampton, and R. CRAIG, Coupling of substructures for dynamic analyses. AIAA Journal, 1968. 6(7): p. 1313-1319.

76. Bladh, R., M. Castanier, and C. Pierre, Component-mode-based reduced order modeling techniques for mistuned bladed disks. Part I: Theoretical models. Journal of Engineering for Gas turbines and Power, 2001. 123(1): p. 89-99.

77. Bladh, R., M. Castanier, and C. Pierre, Component-mode-based reduced order modeling techniques for mistuned bladed disks. Part II: Application. Journal of Engineering for Gas turbines and Power, 2001. 123(1): p. 100-108.

78. Petyt, M., Introduction to finite element vibration analysis. 2010: Cambridge university press.

79. Butland, A. and P. Avitabile, A reduced order, test verified component mode synthesis approach for system modeling applications. Mechanical Systems and Signal Processing, 2010. 24(4): p. 904-921.

80. Yang, M.-T. and J. Griffin, A reduced-order model of mistuning using a subset of nominal system modes. Journal of Engineering for Gas turbines and Power, 2001. 123(4): p. 893-900.

81. Lim, S.-H., et al., Compact, Generalized Component Mode Mistuning Representation for Modeling Bladed Disk Vibration. AIAA Journal, 2007. 45(9): p. 2285-2298. 
82. Yang, M.-T. and J. Griffin, A normalized modal eigenvalue approach for resolving modal interaction. Journal of Engineering for Gas turbines and Power, 1997. 119(3): p. 647-650.

83. Pierre, M.P.C.C., Modeling and Analysis of Mistuned Bladed Disk Vibration:Status and Emerging Directions. JOURNAL OF PROPULSION AND POWER, 2006. 22(2).

84. Petrov, E., K. Sanliturk, and D. Ewins, A new method for dynamic analysis of mistuned bladed disks based on the exact relationship between tuned and mistuned systems. Journal of Engineering for Gas turbines and Power, 2002. 124(3): p. 586-597.

85. Sherman, J. and W.J. Morrison, Adjustment of an inverse matrix corresponding to a change in one element of a given matrix. The Annals of Mathematical Statistics, 1950. 21(1): p. 124-127.

86. Woodbury, M.A., Inverting modified matrices. Memorandum report, 1950. 42: p. 106.

87. Muszyńska, A. and D. Jones, On tuned bladed disk dynamics: Some aspects of friction related mistuning. Journal of Sound and Vibration, 1983. 86(1): p. 107-128.

88. Lin, C.-C. and M.P. Mignolet, Effects of damping and damping mistuning on the forced vibration response of bladed disks. Journal of Sound and Vibration, 1996. 193(2): p. 525-543.

89. Huang, B. and J. Kuang, Mode localization in a rotating mistuned turbo disk with Coriolis effect. International Journal of Mechanical Sciences, 2001. 43(7): p. 1643-1660.

90. Nikolic, M., E. Petrov, and D. Ewins, Coriolis forces in forced response analysis of mistuned bladed disks. Journal of turbomachinery, 2007. 129(4): p. 730-739.

91. Bladh, R., M. Castanier, and C. Pierre, Effects of multistage coupling and disk flexibility on mistuned bladed disk dynamics. Journal of Engineering for Gas turbines and Power, 2003. 125(1): p. 121-130.

92. Sinha, A., Reduced-Order Model of a Mistuned Multi-Stage Bladed Rotor. International Journal of Turbo and Jet Engines, 2008. 25(3): p. 145.

93. Song, S.H., M.P. Castanier, and C. Pierre. System identification of multistage turbine engine rotors. 2007. ASME.

94. Hansteen, O.E. and K. Bell, On the accuracy of mode superposition analysis in structural dynamics. Earthquake Engineering \& Structural Dynamics, 1979. 7(5): p. 405-411.

95. Tran, D.-M., Component mode synthesis methods using interface modes. Application to structures with cyclic symmetry. Computers \& Structures, 2001. 79(2): p. 209-222.

96. Jacquelin, E., et al., Polynomial Chaos Expansion and Steady-State Response of a Class of Random Dynamical Systems. Journal of Engineering Mechanics, 2014.

97. Kundu, A., et al., A hybrid spectral and metamodeling approach for the stochastic finite element analysis of structural dynamic systems. Computer Methods in Applied Mechanics and Engineering, 2014. 270: p. 201-219.

98. Daouk, S., et al., Uncertainties in structural dynamics: overview and comparative analysis of methods. Mechanics \& Industry, 2015. 16(4): p. 404.

99. Kundu, A., S. Adhikari, and M. Friswell, Stochastic finite elements of discretely parameterized random systems on domains with boundary uncertainty. International Journal for Numerical Methods in Engineering, 2014. 100(3): p. 183-221.

100. Rubinstein, R.Y. and D.P. Kroese, Simulation and the Monte Carlo method. Vol. 707. 2011: John Wiley \& Sons.

101. Li, J., et al. Experimental Monte Carlo mistuning assessment of bladed disk vibration using forcing variations. in Proceedings of the 47th AIAA/ASME/ASCE/AHS/ASC Structures, 
Structural dynamics, and Materials Conference. 2006.

102. Chan, Y.J., Variability of blade vibration in mistuned bladed discs. 2009, Imperial College London (University of London).

103. Papadrakakis, M. and A. Kotsopulos, Parallel solution methods for stochastic finite element analysis using Monte Carlo simulation. Computer Methods in Applied Mechanics and Engineering, 1999. 168(1): p. 305-320.

104. Chan, Y.-J. and D. Ewins, The amplification of vibration response levels of mistuned bladed disks: its consequences and its distribution in specific situations. Journal of Engineering for Gas Turbines and Power, 2011. 133(10): p. 102502.

105. Neuringer, J.L., et al., Fatigue life estimates of mistuned blades via a stochastic approach. AIAA Journal, 1980. 18(3): p. 318-323.

106. Mignolet, M.P., W. Hu, and I. Jadic, On the forced response of harmonically and partially mistuned bladed disks. Part II: partial mistuning and applications. International Journal of Rotating Machinery, 2000. 6(1): p. 43-56.

107. Mignolet, M.P., W. Hu, and I. Jadic, On the forced response of harmonically and partially mistuned bladed disks. Part I: Harmonic mistuning. International Journal of Rotating Machinery, 2000. 6(1): p. 29-41.

108. Sinha, A. Statistics of the peak maximum amplitude of the forced response of a mistuned bladed disk. 2005. ASME.

109. Sanliturk, K., M. Imregun, and D. Ewins. Statistical analysis of random mistuning of bladed assemblies. in INSTITUTION OF MECHANICAL ENGINEERS CONFERENCE PUBLICATIONS. 1992. MEDICAL ENGINEERING PUBLICATIONS LTD.

110. Caflisch, R.E., Monte carlo and quasi-monte carlo methods. Acta numerica, 1998. 7: p. 1-49.

111. Blatman, G., B. Sudret, and M. Berveiller, Quasi random numbers in stochastic finite element analysis. Mécanique \& Industries, 2007. 8(03): p. 289-297.

112. Imbens, G.W. and T. Lancaster, Efficient estimation and stratified sampling. Journal of Econometrics, 1996. 74(2): p. 289-318.

113. Helton, J.C. and F.J. Davis, Latin hypercube sampling and the propagation of uncertainty in analyses of complex systems. Reliability Engineering \& System Safety, 2003. 81(1): p. 23-69.

114. Kewlani, G., J. Crawford, and K. Iagnemma, A polynomial chaos approach to the analysis of vehicle dynamics under uncertainty. Vehicle System Dynamics, 2012. 50(5): p. 749-774.

115. Melchers, R., Importance sampling in structural systems. Structural safety, 1989. 6(1): p. 3-10.

116. Law, A.M., W.D. Kelton, and W.D. Kelton, Simulation modeling and analysis. Vol. 2. 1991: McGraw-Hill New York.

117. Madsen, H.O., S. Krenk, and N.C. Lind, Methods of structural safety. 2006: Courier Corporation.

118. Cai, G. and I. Elishakoff, Refined second-order reliability analysis. Structural Safety, 1994. 14(4): p. 267-276.

119. Cizelj, L., B. Mavko, and H. Riesch-Oppermann, Application of first and second order reliability methods in the safety assessment of cracked steam generator tubing. Nuclear Engineering and Design, 1994. 147(3): p. 359-368.

120. Wu, Y.-T., H. Millwater, and T. Cruse, Advanced probabilistic structural analysis method for implicit performance functions. AIAA journal, 1990. 28(9): p. 1663-1669. 
121. Yamazaki, F., et al., Neumann expansion for stochastic finite element analysis. Journal of Engineering Mechanics, 1988. 114(8): p. 1335-1354.

122. Xiu, D. and G.E. Karniadakis, The Wiener--Askey polynomial chaos for stochastic differential equations. SIAM journal on scientific computing, 2002. 24(2): p. 619-644.

123. Sinha, A. Computation of the statistics of forced response of a mistuned bladed disk assembly via polynomial chaos. 2003. ASME.

124. Belytschko, T., Y.Y. Lu, and L. Gu, Element - free Galerkin methods. International journal for numerical methods in engineering, 1994. 37(2): p. 229-256.

125. Baik, S., M.P. Castanier, and C. Pierre. Mistuning sensitivity prediction of bladed disks using eigenvalue curve veerings. in Proceedings of the 9th National Turbine Engine High Cycle Fatigue Conference. 2004.

126. Au, S.-K. and J.L. Beck, Estimation of small failure probabilities in high dimensions by subset simulation. Probabilistic Engineering Mechanics, 2001. 16(4): p. 263-277.

127. Lukáš, P., M. Klesnil, and J. Polak, High cycle fatigue life of metals. Materials Science and Engineering, 1974. 15(2): p. 239-245.

128. Metropolis, N., et al., Equation of state calculations by fast computing machines. The journal of chemical physics, 2004. 21(6): p. 1087-1092.

129. Hastings, W.K., Monte Carlo sampling methods using Markov chains and their applications. Biometrika, 1970. 57(1): p. 97-109.

130. Ching, J., S.-K. Au, and J.L. Beck, Reliability estimation for dynamical systems subject to stochastic excitation using subset simulation with splitting. Computer methods in applied mechanics and engineering, 2005. 194(12): p. 1557-1579.

131. Au, S., J. Ching, and J. Beck, Application of subset simulation methods to reliability benchmark problems. Structural Safety, 2007. 29(3): p. 183-193.

132. Kielb, R.E., et al. Probabilistic analysis of mistuned bladed disks and blisks with aerodynamic and FMM structural coupling. in Proceedings of the 9th national turbine engine HCF conference. 2004.

133. Aquilante, F., et al., Cholesky Decomposition-Based Multiconfiguration Second-Order Perturbation Theory (CD-CASPT2): Application to the Spin-State Energetics of Colll (diiminato)(NPh). Journal of Chemical Theory and Computation, 2008. 4(5): p. 694-702.

134. Choi, J., et al., Design and implementation of the ScaLAPACK LU, QR, and Cholesky factorization routines. Scientific Programming, 1996. 5(3): p. 173-184.

135. Ozer, M.B. and T.J. Royston, Application of Sherman-Morrison matrix inversion formula to damped vibration absorbers attached to multi-degree of freedom systems. Journal of sound and vibration, 2005. 283(3): p. 1235-1249.

136. Level, P., et al., On a direct inversion of the impedance matrix in response reanalysis. Communications in numerical methods in engineering, 1996. 12(3): p. 151-159.

137. Sanliturk, K.Y., et al., Friction damper optimization: simulation of rainbow tests. Journal of Engineering for Gas Turbines and Power, 2001. 123(4): p. 930-939.

138. Petrov, E., et al., Quantitative prediction of the effects of mistuning arrangement on resonant response of a practical turbine bladed disc. ROLLS ROYCE PLC-REPORT-PNR, 2000.

139. Yuan, J., et al., Numerical assessment of using Sherman-Morrison, Neumann expansion techniques for stochastic analysis of mistuned bladed disc systems, in Proceeding of ASME Turbo Expo. 2015: Montral, Canada. 
140. AQUINO, R.J., Stochastic Finite Element Modeling and Its Applications in Heat Conduction and Elastostatics Problems. Swansea University UK, 2009.

141. Wang, X., S. Cen, and C. Li, Generalized Neumann Expansion and Its Application in Stochastic Finite Element Methods. Mathematical Problems in Engineering, 2013. 2013.

142. Sinou, J.-J. and E. Jacquelin, Influence of Polynomial Chaos expansion order on an uncertain asymmetric rotor system response. Mechanical Systems and Signal Processing, 2015. 50: p. 718-731. 